\title{
ON THE EXISTENCE AND REGULARITY OF FUNDAMENTAL DOMAINS WITH LEAST BOUNDARY AREA
}

\author{
JAIGYOUNG CHOE
}

\begin{abstract}
Introduction
Let $M$ be a three-dimensional compact smooth Riemannian manifold. Let $\Phi_{0}$ be the set of all fundamental domains of $M$ with Lipschitz boundary in $\tilde{M}$, the universal covering space of $M$. Then it is a question of basic interest to see whether one can find a fundamental domain in $\Phi_{0}$ with least boundary area among all fundamental domains in $\Phi_{0}$. Moreover, passing to subfamilies of $\Phi_{0}$, one can ask similar questions: Let $\Phi_{1}$ be the subfamily of $\Phi_{0}$ consisting of all fundamental domains of $M$ which are homeomorphic to an open ball, and let $\Phi_{2}$ be the subfamily of $\Phi_{1}$ consisting of all fundamental domains of $M$ whose closures are homeomorphic to a closed ball. Can one find a fundamental domain in $\Phi_{1}$, or $\Phi_{2}$, whose boundary area (counting multiplicity) is the minimum among all fundamental domains in $\Phi_{1}$, or $\Phi_{2}$ ? These problems were proposed by Michael H. Freedman.

In this paper we answer the first problem, the case of $\Phi_{0}$, in the affirmative (Theorem 3). We then discuss the second problem, the case of $\Phi_{1}$, and derive an affirmative answer under the assumption that $M$ is irreducible, that is, every embedded sphere in $M$ bounds a ball (Theorem 5 ). The third problem, the case of $\Phi_{2}$, remains open. Besides the existence of minimizing fundamental domains in $\Phi_{0}$ and $\Phi_{1}$, we also obtain the regularity of the boundaries of these minimizing fundamental domains (Theorem 4). If we define a spine to be a subset of $M$ whose complement in $M$ is homeomorphic to an open ball, then the second problem is equivalent to finding an area minimizing spine of $M$.

For a two-dimensional compact Riemannian manifold $M^{2}$ the problem is much simpler to solve and easier to visualize. In fact, any fundamental domain of $M^{2}$ with least boundary length among all fundamental domains is always homeomorphic to an open disk. Furthermore the boundary of a minimizing fundamental domain consists of geodesic segments of $\tilde{M}^{2}$ meeting each other at $120^{\circ}$ angles, and the number of edges and vertices are both $6-6 \chi(M)$
\end{abstract}

Received March 4, 1987, and, in revised form, January 13, 1988. 
(Proposition). The simplest example is the flat torus $T^{2}$. A minimizing fundamental domain of $T^{2}$ is not the square of Figure 1 but the hexagon of Figure 2 (see Appendix, 1). This is because a triple point is area minimizing under Lipschitz map without counting multiplicity whereas a quadruple point is not.

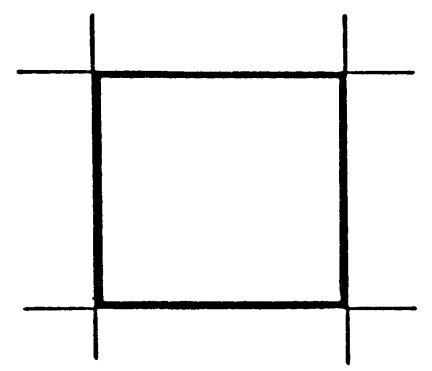

FIGURE 1

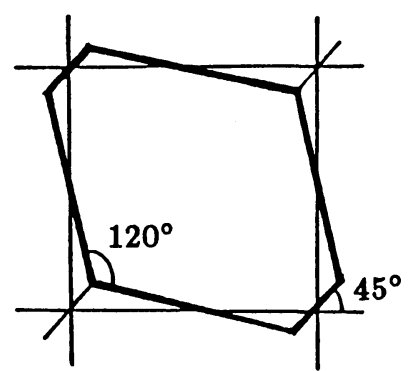

FIGURE 2

The methods used in this paper are those of geometric measure theory. In showing the existence of a minimizing fundamental domain for the first problem, we consider the characteristic functions of fundamental domains and use the compactness of functions of bounded variation. The existence for the second problem follows from the compactness of varifolds, viewing the boundaries of fundamental domains as 2-varifolds.

Throughout this paper we apply the cutting and pasting process extensively. In this process, however, in order not to change the topology of fundamental domains, we must assume that $M$ is irreducible for the second problem. An example, the standard $S^{2} \times S^{1}$, indicates that irreducibility is necessary: A fundamental domain $S^{2} \times(0,1)$ in the universal covering space $S^{2} \times \mathbf{R}^{1}$ of $S^{2} \times S^{1}$ has least boundary area among the elements of $\Phi_{0}$ (see Appendix, $2)$. But $\partial\left(S^{2} \times(0,1)\right)$ is also the varifold limit of $\left\{\partial F_{k}\right\}$, where the $F_{k}$ 's are fundamental domains in $\Phi_{1}$ obtained by cutting out a slanted rod with thickness $\varepsilon_{k}, \varepsilon_{k} \rightarrow 0$ as $k \rightarrow \infty$, which connects $S^{2} \times\{0\}$ to $S^{2} \times\{1\}$, translating and pasting the rod to $S^{2} \times(0,1)$ along $S^{2} \times\{1\}$ (Figure 3$)$. $\left\{F_{k}\right\}$ is also a minimizing sequence in $\Phi_{2}$.

The main difficulty lies in controlling unbounded fundamental domains. Indeed if $\tilde{M}$ is noncompact, then the fundamental domains of $M$ may be unbounded. Moreover, since an unbounded thin spike may have arbitrarily small boundary area, we can have a minimizing sequence of fundamental domains which are unbounded in $\tilde{M}$. This bad minimizing sequence is to be replaced by a uniformly bounded one by applying a cutting and pasting process (Theorem 2). To do so, we should verify that both $\Phi_{0}$ and $\Phi_{1}$ are closed 


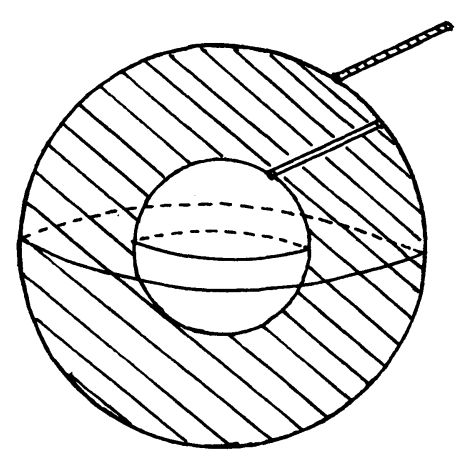

FIGURE 3

under cutting and pasting process, and that the boundary area of fundamental domains does not increase substantially after cutting and pasting. These are verified through an appropriate adaptation of [4] to our setting. This adaptation requires a great deal of care.

Once we have uniform boundedness of the minimizing sequence we can get the desired regularity results for the first problem as in [13]. For the second problem, we need to modify carefully the arguments in [13] to preserve the topology of fundamental domains. Thus we show that the projection into $M$ of the boundary of a minimizing fundamental domain consists of minimal surfaces meeting each other at equal $\left(120^{\circ}\right)$ angles along Hölder continuously differentiable curves, like compound soap films, and four such curves meet each other at isolated points at which six sheets of minimal surfaces meet at equal angles. It should be mentioned that from [8] we can obtain analyticity of the singular curve in case $\tilde{M}$ is isometric to a Euclidean 3 -space $\mathbf{R}^{3}$, and higher regularity in general.

Now we mention two outstanding problems: (i) What is a fundamental domain of a flat cubic torus $\mathbf{T}^{3}$ with least boundary area? (See Appendix 3.) (ii) If the curvature of $M$ is nonpositive, does the minimizing fundamental domain of $M$ in $\Phi_{0}$ or $\Phi_{1}$ also belong to $\Phi_{2}$ ? Is the minimizer star-shaped?

Finally, we wish to express our sincere gratitude to Richard M. Schoen, who introduced us to geometry and analysis. Also we would like to thank Michael H. Freedman and Leon Simon for their interest and helpful discussions.

\section{Terminology}

In general, we will use the definitions and notation of [7] and [13] throughout. 
(1) Let $M$ be a three-dimensional compact smooth Riemannian manifold, $\tilde{M}$ the universal covering space of $M$, and $p$ the projection map from $\tilde{M}$ onto $M$.

A fundamental domain of $M$ is an open set $F$ in $\tilde{M}$ satisfying

$$
\operatorname{Vol}(F)=\operatorname{Vol}(p(F))=\operatorname{Vol}(M) .
$$

Let $\Phi_{0}$ denote the set of all fundamental domains of $M$ with Lipschitz boundary and let $\Phi_{1}$ denote the set of all fundamental domains in $\Phi_{0}$ which are homeomorphic to an open ball. Then, for any $F \in \Phi_{1}, M \sim p(\partial F)$ is homeomorphic to an open ball. A subset $S$ of $M$ will be called a spine of $M$ if $M \sim S$ is homeomorphic to an open ball. Note that, for any $F \in \Phi_{1}, p(\partial F)$ is a spine of $M$ and conversely, for any Lipschitz spine we can find $F \in \Phi_{1}$ with $p(\partial F)=S$.

A fundamental domain $F \in \Phi_{1}$ will be said to be reducible if there exists a proper subset $S$ of $p(\partial F)$ such that $S$ has Lipschitz boundary and $M \sim S$ is still homeomorphic to an open ball. Let $\Phi$ denote the set of all fundamental domains in $\Phi_{1}$ which are not reducible.

A fundamental domain $F \in \Phi$ will be said to be adequate if $\bar{F}$ is homeomorphic to a closed ball, otherwise $F$ will be said to be inadequate.

Consider the subset $R_{F}$ of $\partial F$ which consists of $q \in \partial F$ with the property that there is an $r_{q}>0$ such that for any geodesic ball $G_{r}(p(q))$ with center $p(q)$ and radius $r<r_{q}, p(F) \cap G_{r}(p(q))$ consists of two components only. Each component of $R_{F}$ will be called a face of $F$. Any subset of $\partial F \sim R_{F}$ which is homeomorphic to an open interval $(0,1)$ will be called a multiple curve of $F$. Any point of $\partial F \sim R_{F}$ at which at least three distinct multiple curves of $F$ meet each other will be called a multiple point of $F$.

For $F \in \Phi_{0}, \delta F$ denotes $p(\partial F)$. The image under $p$ of face, multiple curve, or multiple point of $F$ will be called face, multiple curve, or multiple point of $\delta F$ respectively.

For any set $K \subset M$, define $\tilde{K}=\{x \in \tilde{M}: p(x) \in K\}$.

(2) $B^{n}(p, r)$ and $U^{n}(p, r)$ will denote respectively the closed and open geodesic balls with radius $r$ and center $p$ in $M^{n}$ or $\mathbf{R}^{n}$. $B, B^{\circ}$ will denote $B^{3}(0,1), U^{3}(0,1)$ in $\mathbf{R}^{3}$ respectively.

We define $D=\left\{x \in \mathbf{R}^{2}:|x|<1\right\}$.

(3) For each $r>0$ we define $\mu_{r}: \mathbf{R}^{n} \rightarrow \mathbf{R}^{n}, \mu_{r}(x)=r x, x \in \mathbf{R}^{n}$, and for each $p \in \mathbf{R}^{n}$ we define $\tau_{p}: \mathbf{R}^{n} \rightarrow \mathbf{R}^{n}, \tau_{p}(x)=x-p, x \in \mathbf{R}^{n}$.

(4) We say that $S$ is area minimizing in an open set $U \subset M^{n}$ under a Lipschitz map provided

$$
H^{m}(S) \leq H^{m}(\phi(S))
$$


whenever $\phi$ is a Lipschitz map on $M^{n}$ such that $\phi$ maps $U$ into $U$ and leaves $M \sim U$ fixed, where $H^{m}$ denotes the $m$-dimensional Hausdorff measure.

A varifold $V$ in $M^{n}$ is said to be area minimizing under diffeomorphism provided

$$
\mathbf{M}(V) \leq \mathbf{M}\left(\phi_{\#} V\right)
$$

whenever $\phi$ is a diffeomorphism of $M^{n}$, where $M$ denotes the mass.

(5) Define $Y^{1} \subset \mathbf{R}^{2}$ to be a union of three half-lines joining at the origin with $120^{\circ}$ angles to each other. Define $Y \subset \mathbf{R}^{3}$ by $Y=\left(Y^{1} \times \mathbf{R}^{1}\right) \cap B^{3}(0,1)$ (see Figure 4).

Define $T \subset \mathbf{R}^{3}$ as the intersection with $B^{3}(0,1)$ of an infinite cone from the origin through the 1-skeleton of a regular tetrahedron with its center of mass at the origin (see Figure 5).

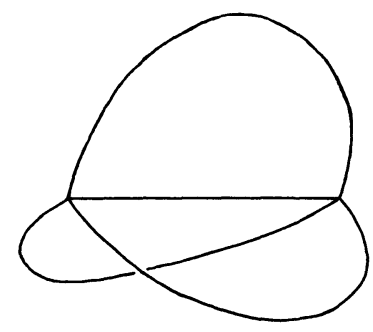

FIGURE 4

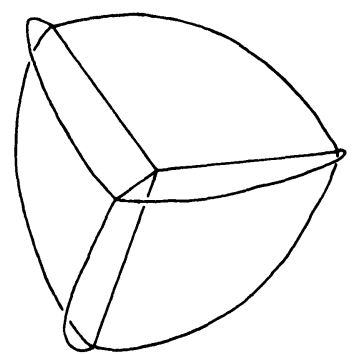

FIGURE 5

(6) For $S$ a 2-rectifiable set, define $\sigma(S)$ as the singular set of $S$, that is, $\sigma(S)$ is the set of points in $S$ at which there is no approximate tangent plane to $S$ in $G(3,2)$, or $\Theta^{2}\left(H^{2}{ }_{\llcorner} S, x\right)$ is not one. Define $\sigma_{Y}(S) \subset \sigma(S)$ as the points of density $3 / 2$, and $\sigma_{T}(S) \subset \sigma(S)$ as the points of density $\pi^{-1} 3 \cos ^{-1}(-1 / 3)$. Define $R(S)=S \sim \sigma(S)$.

If $V$ is a 2-varifold we define $\sigma_{Y}(V)=\sigma_{Y}(\operatorname{spt}\|V\|), \sigma_{T}(V)=\sigma_{T}(\operatorname{spt}\|V\|)$, $R(V)=R(\operatorname{spt}\|V\|)$, and $\sigma(V)=\sigma(\operatorname{spt}\|V\|)$.

(7) Suppose $S$ is a surface homeomorphic to $D$ or $\partial B$. A compound Jordan curve in $S$ is a connected union of a finite number of Jordan curves in $S$. Given a compound Jordan curve $C$ in $S$, a Jordan curve $C_{1} \subset C$ is said to be an individual Jordan curve of $C$ if there exists a set $X \subset S \sim C$ with $X \approx D$ and $\partial X=C_{1}$. In case $S \approx D$, a subset $C_{2}$ of $C$ is said to be an outermost Jordan curve of $C$ if there exists an annular domain $Y \subset S \sim C$ with $C_{2}=\partial Y \sim \partial S$.

(8) Recall that $\Phi_{0}$ consists of fundamental domains of $M$ with Lipschitz boundary. Hence, given $F \in \Phi_{0}$, there exists a family $\left\{S_{i}\right\}_{i \in I}$ of pairwise 
disjoint subsets of $\delta F(=p(\partial F))$ with $\bigcup_{i \in I} \bar{S}_{i}=\delta F$ such that for all $i \in I, S_{i}$ is $C^{1}$ up to its boundary and $\partial S_{i}$ is a Lipschitz curve. Let $X$ be a $C^{1}$ surface in $M$. Then we say that $\delta F$ is transversal to $X$ if $S_{i}$ and $\partial S_{i}$ are transversal to $X$ for all $i \in I$. It follows from Sard's theorem that for almost all $r<i(M)$, the injectivity radius of $M, \delta F$ intersects $\partial B^{3}(p, r)$ transversally and each component of $\delta F \cap \partial B^{3}(p, r)$ is a compound Jordan curve on $\partial B^{3}(p, r)$.

\section{Replacement theorem and rectifiability}

The following theorem guarantees that if $U$ is an open convex subset of $M$, then we can always replace $F \in \Phi_{0}$ (respectively $\Phi$ ) by $\hat{F} \in \Phi_{0}$ (respectively $\Phi)$ in such a way that $\delta \hat{F}$ satisfies an isoperimetric inequality in $U$.

Theorem 1 (Replacement Theorem). (1) Given $U \approx B^{\circ}$ with $C^{1}$ boundary $\partial U$ in $M$, and $F \in \Phi_{0}$ with $\delta F$ intersecting $\partial U$ transversally, there exists $\hat{F} \in \Phi_{0}$ such that

(i) $\delta \hat{F} \sim \bar{U}=\delta F \sim \bar{U}$;

(ii) $\delta \hat{F} \cap \bar{U} \subset \partial U$ and $H^{2}(\delta \hat{F} \cap \bar{U}) \leq c\left(H^{1}(\delta F \cap \partial U)\right)^{2}$ for some $c>0$ depending on $U$.

(2) Suppose $M$ is irreducible. Given $U \approx B^{0}$ with $C^{1}$ boundary $\partial U$ and $F \in \Phi$ with $\delta F$ intersecting $\partial U$ transversally, there exists $\hat{F} \in \Phi$ such that

(i) $\delta \hat{F} \sim U \subset \delta F \sim U$;

(ii) $\delta \hat{F} \cap U$ is a disjoint union of surfaces homeomorphic to $D$; if $U$ is convex, then

(ii) $^{\prime} \delta \hat{F} \cap U$ is a disjoint union of area minimizing surfaces homeomorphic to $D$;

(iii) $H^{2}(\delta \hat{F} \cap U) \leq c\left(H^{1}(\delta \hat{F} \cap \partial U)\right)^{2}$ for some $c>0$ depending on $U$.

For the proof of Theorem 1 we need the following two lemmas.

Lemma 1 (Isoperimetric Inequality). (1) Let $C_{0}$ be a union of compound Jordan curves on a $C^{1}$ surface $S \approx \partial B$ in $M$ and let $Y_{0}, \cdots, Y_{l}$ be open components of $S \sim C_{0}$. Suppose $\max _{0 \leq i \leq l}\left\{H^{2}\left(Y_{i}\right)\right\}=H^{2}\left(Y_{0}\right)$. Then there exists $c>0$ depending on $S$ such that $H^{2}\left(S \sim Y_{0}\right) \leq c\left(H^{1}\left(C_{0}\right)\right)^{2}$.

(2) Let $C$ be a compound Jordan curve on a $C^{1}$ surface $S \approx \partial B$ in $M$ and let $X_{0}, \cdots, X_{n}$ be the open components of $S \sim C$ where every $X_{i}$ is homeomorphic to $D$. Suppose $\max _{0 \leq i \leq n}\left\{H^{2}\left(X_{i}\right)\right\}=H^{2}\left(X_{0}\right)$. Then there exists $c>0$ depending on $S$ such that $H^{2}\left(S \sim X_{0}\right) \leq c\left(H^{1}(C)\right)^{2}$.

Proof of Lemma 1. Croke [5] showed that there exists a constant $c$ depending on $S$ such that whenever $E$ is a region on $S$ with $E \approx D$, then

$$
\min \left\{H^{2}(E), H^{2}(S \sim E)\right\} \leq c\left(H^{1}(\partial E)\right)^{2} .
$$


We prove (2) first. Let $\beta=H^{2}(S)$ and $\alpha=H^{2}\left(X_{0}\right)$. First suppose $\alpha \leq \beta / 2$. Then

$$
\begin{aligned}
H^{2}\left(S \sim X_{0}\right) & =\beta-\alpha \leq \frac{\beta^{2}}{4 \alpha}=c\left(\frac{1}{2} \sqrt{\frac{\alpha}{c} \frac{\beta}{\alpha}}\right)^{2} \\
& =c\left(\frac{1}{2} \sum_{i=0}^{n} \frac{1}{\alpha} \sqrt{\frac{\alpha}{c}} H^{2}\left(X_{i}\right)\right)^{2} \\
& \leq c\left(\frac{1}{2} \sum_{i=0}^{n} \frac{1}{H^{2}\left(X_{i}\right)} \sqrt{\frac{H^{2}\left(X_{i}\right)}{c}} H^{2}\left(X_{i}\right)\right)^{2} \\
& \leq c\left(\frac{1}{2} \sum_{i=0}^{n} H^{1}\left(\partial X_{i}\right)\right)^{2}=c\left(H^{1}(C)\right)^{2} .
\end{aligned}
$$

In case $\alpha>\beta / 2$,

$$
H^{2}\left(S \sim X_{0}\right) \leq c\left(H^{1}\left(\partial X_{0}\right)\right)^{2} \leq c\left(H^{1}(C)\right)^{2} .
$$

(1) Suppose $Y \subset S$ is a multiply connected open set with $H^{2}(Y) \leq \beta / 2$. First, assume there exists a component $Z$ of $S \sim Y$ such that $Z \approx \bar{D}$ and $H^{2}(Z)>\beta / 2$. Then

$$
H^{2}(Y) \leq H^{2}(S \sim Z) \leq c\left(H^{1}(\partial Z)\right)^{2} \leq c\left(H^{1}(\partial Y)\right)^{2} .
$$

Second, assume $H^{2}\left(Z_{i}\right) \leq \beta / 2$ for each component $Z_{i}$ of $S \sim Y$. Then

$$
H^{2}(Y) \leq \sum_{i} H^{2}\left(Z_{i}\right) \leq \sum_{i} c\left(H^{1}\left(\partial Z_{i}\right)\right)^{2} \leq c\left(\sum_{i} H^{1}\left(\partial Z_{i}\right)\right)^{2}=c\left(H^{1}(\partial Y)\right)^{2} .
$$

Therefore, in either case, $H^{2}(Y) \leq c\left(H^{1}(\partial Y)\right)^{2}$.

Let $\alpha_{0}=H^{2}\left(Y_{0}\right)$ and suppose $\alpha_{0} \leq \beta / 2$. Then, as in (2), we have

$$
H^{2}\left(S \sim Y_{0}\right) \leq c\left(\frac{1}{2} \sum_{i=0}^{l} \frac{1}{H^{2}\left(Y_{i}\right)} \sqrt{\frac{H^{2}\left(Y_{i}\right)}{c}} H^{2}\left(Y_{i}\right)\right)^{2} .
$$

Since $H^{2}\left(Y_{i}\right) \leq c\left(H^{1}\left(\partial Y_{i}\right)\right)^{2}$ for all $i=1, \cdots, l$, we have

$$
H^{2}\left(S \sim Y_{0}\right) \leq c\left(\frac{1}{2} \sum_{i=0}^{l} H^{1}\left(\partial Y_{i}\right)\right)^{2}=c\left(H^{1}\left(C_{0}\right)\right)^{2} .
$$

If $\alpha_{0}>\beta / 2$, then

$$
H^{2}\left(S \sim Y_{0}\right)=\sum_{j} H^{2}\left(Z_{j}\right)
$$


where $\left\{Z_{j}\right\}$ is the set of all components of $S \sim Y_{0}$. Since $Z_{j} \approx \bar{D}$ and $H^{2}\left(Z_{j}\right) \leq \beta / 2$, we get

$$
H^{2}\left(S \sim Y_{0}\right) \leq \sum_{j} c\left(H^{1}\left(\partial Z_{j}\right)\right)^{2} \leq c\left(\sum_{j} H^{1}\left(\partial Z_{j}\right)\right)^{2} \leq c\left(H^{1}\left(C_{0}\right)\right)^{2} .
$$

Lemma 2. (1) Let $F \in \Phi_{0}$, and assume $U \approx B^{\circ}$ has $C^{1}$ boundary $\partial U$ which is transversal to $\delta F$. Then there exists $\hat{F} \in \Phi_{0}$ with $\delta \hat{F} \sim \bar{U}=\partial F \sim \bar{U}$, $\delta \hat{F} \cap \bar{U} \subset \partial U$ such that $\partial U \sim(\delta \hat{F} \cap \bar{U})$ is a component of $\partial U \sim \delta F$.

(2) Assume $M$ is irreducible, $F \in \Phi$, and $E$ is a piecewise $C^{1}$ surface in $M$ with $E \approx D$. If $\delta F \cap E$ is a compound Jordan curve disjoint from $\partial E$, then there exist $\hat{F} \in \Phi$ and a set $W \approx B^{\circ}$ in $M$ such that $E \subset \partial W$, $\delta F \cap \partial W=\delta F \cap E$, and $\delta \hat{F} \subset(\delta F \sim W) \cup E$.

Proof of Lemma 2. (1) Let $Y$ be a set in $F$ such that $p(Y)$ is a component of $\partial U \sim \delta F$. Let $\hat{U} \subset \tilde{M}$ be a component of $\tilde{U}$ with $Y \subset \partial \hat{U}$. Define $\hat{F}=(F \sim \overline{\tilde{U}}) \cup \hat{U} \cup Y$. Then we see that $\hat{F}$ is a fundamental domain of $M$ in $\Phi_{0}$. Obviously $\delta \hat{F} \sim \bar{U}=\delta F \sim \bar{U}$ and $\delta \hat{F} \cap \bar{U}=\partial U \sim p(Y)$.

(2) Since $\delta F \cap \partial E=\varnothing$ and $M \sim \delta F \approx B^{\circ}$, there exists a set $J \approx \bar{D}$ in $M$ such that $\delta F \cap J=\varnothing, \partial J=\partial E$, and $J \cap \bar{E}=\partial E$. Then $J \cup E$ is homeomorphic to a sphere, and hence by the irreducibility hypothesis there exists a set $W \approx B^{\circ}$ in $M$ with $\partial W=J \cup E$. Note that $\delta F \cap \partial W=\delta F \cap E$. Now let $W_{1}$ be the component of $\tilde{W}$ with $\partial W_{1} \supset|F \cap \tilde{J}|$, and define $F_{1} \in \Phi_{0}$ by $F_{1}=(F \sim \tilde{W}) \cup W_{1} \cup\left(F \cap \partial W_{1}\right)$. Then, since each component of $F \sim p^{-1}(\partial W)$ is homeomorphic to $B^{\circ}$, so is each component of $F_{1}$. Let us assume that $U_{0}$ is the component of $F_{1}$ containing $F \cap \partial W_{1}$, and $U_{1}, \cdots, U_{m}$ are the remaining components of $F_{1}$. Then it is easy to see $\partial W \cap p\left(\bar{U}_{i}\right) \approx D, i=1, \cdots, m$. Hence we can find $U_{i, 0}$, a component of $p^{-1}\left(p\left(U_{i}\right)\right)$, with $\partial W_{1} \cap \bar{U}_{i, 0} \approx D$ for each $i=1, \cdots, m$. We thus paste each $U_{i, 0}$ to $U_{0}$ along $\partial W_{1} \cap \bar{U}_{i, 0}$ to get a fundamental domain $\hat{F} \in \Phi$ with $\delta \hat{F} \subset(\delta F \sim W) \cup E$, and hence the proof is complete.

Proof of Theorem 1. (1) Let $Y$ be a set in $F$ such that $p(Y)$ is a component of $\partial U \sim \delta F$ with $H^{2}(p(Y))=\max \left\{H^{2}\left(Y_{i}\right)\right\}$, where the maximum is taken over all components $Y_{i}$ of $\partial U \sim \delta F$. Then Lemma 1(1) and Lemma 2(1) prove (1).

(2) Let $K_{i}, i=1, \cdots, l$, be the components of $\delta F \cap \bar{U}$ and let $C_{i j}, j=$ $1, \cdots, m_{i}$, be the disjoint compound Jordan curves such that $K_{i} \cap \partial U=$ $\bigcup_{j=1}^{m_{i}} C_{i j}$ for each $i$. Finally let $X_{i j k} \subset \partial U, 1 \leq k \leq n_{i j}$, be such that $X_{i j k} \approx D, \partial U \sim C_{i j}=\bigcup_{k=1}^{n_{i j}} X_{i j k}$, for each $i$ and $j$. Renumbering if necessary, one can assume $\max _{1 \leq k \leq n_{i j}}\left\{H^{2}\left(X_{i j k}\right)\right\}=H^{2}\left(X_{i j 1}\right)$ for each $i$ and $j$. Define $Y_{i j}=\partial U \sim \bar{X}_{i j 1}$. Then $Y_{i j} \approx D$. Suppose $Y_{a b} \cap Y_{c d} \neq \varnothing$ for some $1 \leq a, c \leq l$, 
$1 \leq b \leq m_{a}$, and $1 \leq d \leq m_{c}$. Since $C_{a b} \cap C_{c d}=\varnothing$, we have three possibilities:

$$
Y_{a b} \subset Y_{c d}, \quad Y_{a b} \supset Y_{c d}, \quad Y_{a b} \cup Y_{c d}=\partial U .
$$

The third case is not possible since otherwise we would get two contradictory conclusions: $H^{2}\left(X_{a b 1}\right)<H^{2}\left(X_{c d 1}\right)$ and $H^{2}\left(X_{a b 1}\right)>H^{2}\left(X_{c d 1}\right)$. The first two cases give us a partial ordering in the family $\left\{C_{i j}\right\}_{1 \leq i \leq l, 1 \leq j \leq m_{i}}$ in a standard way, i.e., $C_{a b}<C_{c d}$ if and only if $Y_{a b} \subset Y_{c d}$. Hence by choosing a minimal element successively from $\left\{C_{i j}\right\}$ one can get an ordered family $\left\{C_{\alpha}\right\}_{0 \leq \alpha \leq n}$ such that $\left\{C_{\alpha}\right\}_{0 \leq \alpha \leq n}=\left\{C_{i j}\right\}_{1 \leq i \leq l, 1 \leq j \leq m_{i}}$ and $C_{\alpha}<C_{\beta}$ only if $\alpha<\beta$. Define $Y_{\alpha}=Y_{i j}$ if $C_{\alpha}=C_{i j}$.

Let $\theta=\min _{0 \leq \alpha \neq \beta \leq n}\left\{\operatorname{dist}\left(C_{\alpha}, C_{\beta}\right)\right\}$. Then we can find $Z_{0} \subset \partial U$ such that $Z \approx D, Z_{0} \supset \bar{Y}_{0}$, and $H D\left(Z_{0}, Y_{0}\right)<\theta$. We thus apply Lemma 2 with $Z_{0}$ in place of $E$, to give $\hat{F} \in \Phi$ and a set $W_{0} \approx B^{\circ}$ such that $Z_{0} \subset \partial W_{0}$, $\delta F \cap \partial W_{0}=\delta F \cap Z_{0}$, and $\delta \hat{F} \subset\left(\delta F \sim W_{0}\right) \cup Z_{0} . W_{0}$ plays the role of eliminating the piece of $\delta F$ which lies either inside $U$ or outside $U$. Hence we consider the following two cases:

Case 1. $W_{0}$ eliminates the piece of $\delta F$ which lies outside $U$, i.e., there is an open set $V_{0}$ containing $Y_{0}$ such that $(\delta F \sim \delta \hat{F}) \cap V_{0} \subset M \sim U$. Taking points of $\delta \hat{F} \cap \bar{Y}_{0}$ into $U$ we obtain $\delta F_{1}, F_{1} \in \Phi$, with $\delta F_{1} \cap \partial U=(\delta \hat{F} \cap \partial U) \sim \bar{Y}_{0}$ $\left(\subset(\delta F \cap \partial U) \sim C_{0}\right)$. Define $U_{1}=U$.

Case 2. $W_{0}$ eliminates the piece of $\delta F$ which lies inside $U$, i.e., there is an open set $V_{0}$ containing $Y_{0}$ such that $(\delta F \sim \delta \hat{F}) \cap V_{0} \subset U$. Holding the set $\delta \hat{F} \sim Y_{0}$ fixed, and taking points of $\delta \hat{F} \cap Y_{0}$ into $U$ we obtain $\delta F_{1}, F_{1} \in \Phi$, with $\delta F_{1} \cap \partial U \subset \delta F \cap \partial U$. Note that if $K_{0}$ is the component of $\delta F_{1} \cap \bar{U}$ containing $C_{0}$, then $K_{0} \sim C_{0}=\bigcup_{i=1}^{m_{1}} Z_{1, i}$, where $Z_{1, i} \approx D$ and $\bigcup_{i=1}^{m_{1}} \partial Z_{1, i}=C_{0}$. Thus we can find an open set $U_{1} \subset U$ such that $U_{1} \approx B^{\circ}$ and $\delta F_{1} \cap\left(U \sim U_{1}\right)=$ $\bigcup_{i=1}^{m_{1}} Z_{i, 1}$. Hence $\delta F_{1} \cap \partial U_{1}=\left(\delta F_{1} \cap \partial U\right) \sim C_{0} \subset \delta F \cap \partial U \sim C_{0}$.

In either case, note that $\delta F_{1} \cap \partial U_{1}$ has fewer components than $\delta F \cap \partial U$, more precisely, $\delta F_{1} \cap \partial U_{1} \subset(\delta F \cap \partial U) \sim C_{0}$. We also have $\delta F_{1} \sim U \subset \delta F \sim$ $U$.

Now that we "eliminated" $C_{0}$ we again apply the same argument (cutting and slight perturbation) to $\delta F_{1} \cap U_{1}$ to eliminate the remaining $C_{\alpha}$ 's. First $\delta F_{1} \cap \partial U_{1}$ can be viewed as a subsequence of $\delta F \cap \partial U$. Let $C_{\alpha_{1}}$ be the first element of this subsequence. Clearly $C_{\alpha_{1}} \neq C_{0}$. Define $Y_{\alpha_{1}, 0} \subset \partial U_{1}$ by

$$
Y_{\alpha_{1}, 0}= \begin{cases}\left(Y_{\alpha_{1}} \sim\left(\partial U \sim \partial U_{1}\right)\right) \cup\left(\partial U_{1} \sim \partial U\right) & \text { if } Y_{\alpha_{1}} \supset Y_{0} \\ Y_{\alpha_{1}} & \text { if } Y_{\alpha_{1}} \cap Y_{0}=\varnothing .\end{cases}
$$

Then $Y_{\alpha_{1}, 0} \approx D$ and hence we can find $Z_{1} \subset \partial U_{1}$ such that $Z_{1} \approx D, Z_{1} \supset$ $\bar{Y}_{\alpha_{1}, 0}$, and $H D\left(Z_{1}, Y_{\alpha_{1}, 0}\right)<\theta$. Now we can apply Lemma 2 , with $Z_{1}$ in place 
of $E$, to give $\hat{F}_{1} \in \Phi$ and a set $W_{1} \approx B^{\circ}$ such that $Z_{1} \subset \partial W_{1}, \delta F_{1} \cap \partial W_{1}=$ $\delta F_{1} \cap Z_{1}$, and $\delta \hat{F}_{1} \subset\left(\delta F_{1} \sim W_{1}\right) \cup Z_{1}$. Here again we consider the following two cases:

Case 1. $\left(\delta F_{1} \sim \delta \hat{F}_{1}\right) \cap V_{1} \subset M \sim U_{1}$ for some open set $V_{1}$ containing $Y_{\alpha_{1}, 0}$. Taking points of $\delta \hat{F}_{1} \cap \bar{Y}_{\alpha_{1}, 0}$ into $U$ we can obtain $\delta F_{2}, F_{2} \in \Phi$ with $\delta F_{2} \cap \partial U_{1}=\left(\delta \hat{F}_{1} \cap \partial U_{1}\right) \sim \bar{Y}_{\alpha_{1}}$. Define $U_{2}=U_{1}$.

Case 2. $\left(\delta F_{1} \sim \delta \hat{F}_{1}\right) \cap V_{1} \subset U_{1}$. In this case holding the set $\delta \hat{F}_{1} \sim Y_{\alpha_{1}}$ fixed, and taking the points of $\delta \hat{F}_{1} \cap Y_{\alpha_{1}}$ into $U_{1}$ we can obtain $\delta F_{2}, F_{2} \in \Phi$, with $\delta F_{2} \cap \partial U_{1} \subset \delta F_{1} \cap \partial U_{1}$. Now we can find an open set $U_{2} \subset U_{1}$ such that $U_{2} \approx B^{\circ}$, and the component of $\delta F_{2} \cap \bar{U}_{1}$ containing $C_{\alpha_{1}}$ is contained in $\bar{U}_{1} \sim U_{2}$. Note that $\delta F_{2} \cap\left(U_{1} \sim U_{2}\right)=\bigcup_{i=1}^{m_{2}} Z_{2, i}$, where $Z_{2, i} \approx D$ and $\bigcup_{i=1}^{m_{2}} \partial Z_{2, i}=C_{\alpha_{1}}$.

In either case, $\delta F_{2} \cap \partial U_{2}$ has fewer components than $\delta F_{1} \cap \partial U_{1}$, i.e., $\delta F_{2} \cap$ $\partial U_{2} \subset\left(\delta F_{1} \cap \partial U_{1}\right) \sim C_{\alpha_{1}}$. Note also that $\delta F_{2} \sim U_{1} \subset \delta F_{1} \sim U_{1}$.

Continuing the above procedure we can obtain for each $j=1, \cdots, n_{0}-1$,

$C_{\alpha_{j}}$ with $0=\alpha_{0}<\alpha_{1}<\cdots<\alpha_{n_{0}-1}, n_{0}-1<n$

(i.e., $\left\{C_{\alpha_{j}}\right\}_{0 \leq j \leq n_{0}-1}$ is a subsequence of $\left\{C_{\alpha}\right\}_{0 \leq \alpha \leq n}$ );

$U_{j+1}$ with $U_{j+1} \approx B^{\circ}$ and $U \supset U_{1} \supset U_{2} \supset \cdots \supset U_{n_{0}}$;

$Y_{\alpha_{j}, 0} \subset \partial U_{j}$ with $Y_{\alpha_{j}, 0} \approx D$, and

$Y_{\alpha_{j}, 0}= \begin{cases}\left(Y_{\alpha_{j}} \sim\left(\partial U \sim \partial U_{j}\right)\right) \cup\left(\partial U_{j} \sim \partial U\right) & \text { if } Y_{\alpha_{j}} \supset Y_{\alpha_{j-1}}, \\ Y_{\alpha_{j}} & \text { if } Y_{\alpha_{j}} \cap Y_{\alpha_{j-1}}=\varnothing ;\end{cases}$

$$
\begin{aligned}
& Z_{j} \subset \partial U_{j} \quad \text { with } Z_{j} \approx D, Z_{j} \supset \bar{Y}_{\alpha_{j}, 0}, \text { and } H D\left(Z_{j}, Y_{\alpha_{j}, 0}\right)<\theta ; \\
& F_{j+1} \in \Phi \text { with } \delta F_{j+1} \sim U_{j} \subset \delta F_{j} \sim U_{j}\left(U_{0}=U, F_{0}=F\right),
\end{aligned}
$$

such that we have the following additional properties:

$$
\begin{aligned}
& C_{\alpha_{j}}, C_{\alpha_{j+1}}, \cdots, C_{\alpha_{n_{0}-1}} \subset \delta F_{j} \cap \partial U_{j} ; \\
& \delta F_{j+1} \cap \partial U_{j+1} \subset\left(\delta F_{j} \cap \partial U_{j}\right) \sim C_{\alpha_{j}}
\end{aligned}
$$

either $U_{j+1}=U_{j}$ or $U_{j+1}$ is a proper subset of $U_{j}$, and

$$
\begin{aligned}
& \delta F_{j+1} \cap\left(U_{j} \sim U_{j+1}\right)=\bigcup_{i=1}^{m_{j+1}} Z_{j+1, i}, \\
& \text { where } Z_{j+1, i} \approx D \text { and } \bigcup_{i=1}^{m_{j+1}} \partial Z_{j+1, i}=C_{\alpha_{j}} .
\end{aligned}
$$

Note that $\partial F_{n_{0}} \cap U_{n_{0}}=\varnothing$, so that

$$
\begin{aligned}
\delta F_{n_{0}} \cap U & =\left[\left(\delta F_{n_{0}} \sim U_{n_{0}}\right) \cup\left(\delta F_{n_{0}} \cap U_{n_{0}}\right)\right] \cap U=\left(\delta F_{n_{0}} \sim U_{n_{0}}\right) \cap U \\
& =\left[\left(\delta F_{n_{0}} \sim U_{n_{0}-1}\right) \cap U\right] \cup\left[\delta F_{n_{0}} \cap\left(U_{n_{0}-1} \sim U_{n_{0}}\right)\right] .
\end{aligned}
$$


Using (2.2) repeatedly we thus have

$$
\begin{aligned}
& \delta F_{n_{0}} \cap U \subset\left[\left(\delta F_{n_{0}-1} \sim U_{n_{0}-1}\right) \cap U\right] \cup\left[\delta F_{n_{0}} \cap\left(U_{n_{0}-1} \sim U_{n_{0}}\right)\right] \\
& =\left[\left(\delta F_{n_{0}-1} \sim U_{n_{0}-2}\right) \cap U\right] \cup \bigcup_{j=0}^{1}\left[\delta F_{n_{0}-j} \cap\left(U_{n_{0}-j-1} \sim U_{n_{0}-j}\right)\right] \\
& \subset\left[\left(\delta F_{n_{0}-2} \sim U_{n_{0}-2}\right) \cap U\right] \cup \bigcup_{j=0}^{1}\left[\delta F_{n_{0}-j} \cap\left(U_{n_{0}-j-1} \sim U_{n_{0}-j}\right)\right] \\
& =\left[\left(\delta F_{n_{0}-2} \sim U_{n_{0}-3}\right) \cap U\right] \cup \bigcup_{j=0}^{2}\left[\delta F_{n_{0}-j} \cap\left(U_{n_{0}-j-1} \sim U_{n_{0}-j}\right)\right] \\
& =\left[\left(\delta F_{1} \sim U\right) \cap U\right] \cup \bigcup_{j=0}^{n_{0}-1}\left[\delta F_{n_{0}-j} \cap\left(U_{n_{0}-j-1} \sim U_{n_{0}-j}\right)\right] \\
& =\bigcup_{j=0}^{n_{0}-1}\left[\delta F_{j+1} \cap\left(U_{j} \sim U_{j+1}\right)\right] .
\end{aligned}
$$

Hence (2.3) gives $\delta F_{n_{0}} \cap U \subset \bigcup_{j=1}^{n_{1}} \bigcup_{i=1}^{m_{\beta_{j}}} Z_{\beta_{j}, i}$, where $\left\{\beta_{1}, \cdots, \beta_{n_{1}}\right\}=\left\{j: U_{j}\right.$ is a proper subset of $\left.U_{j-1}\right\}, n_{1} \leq n_{0}, Z_{k, i}$ is homeomorphic to $D$, and $\bigcup_{i=1}^{m_{k}} \partial Z_{k, i}=C_{\alpha_{k-1}}$. In fact, renumbering $j$ if necessary, one can easily have

$$
\delta F_{n_{\cup}} \cap U=\bigcup_{k=1}^{n_{2}} \bigcup_{i=1}^{m_{k}} Z_{k, i}
$$

where $n_{2} \leq n_{1}$ and $\left\{j: C_{\alpha_{j-1}} \subset \delta F_{n_{0}} \cap \partial U\right\}=\left\{1,2, \cdots, n_{2}\right\}$. This proves (ii) if we let $F_{n_{0}}=\hat{F}$. (2.1) and (2.2) prove (i).

Now we assume $U$ is convex. The next step in the argument involves replacing each $Z_{k, i}$ by an area minimizing surface $\hat{Z}_{k, i} \approx D$ with $\partial \hat{Z}_{k, i}=$ $\partial Z_{k, i}$. Since the family $\left\{\hat{Z}_{k, i}\right\}$ is pairwise disjoint, we can get a fundamental domain $\hat{F} \in \Phi$ such that $\delta \hat{F} \sim U=\delta F_{n_{0}} \sim U$ and

$$
\delta \hat{F} \cap U=\bigcup_{k=1}^{n_{2}} \bigcup_{i=1}^{m_{k}} \hat{Z}_{k, i}
$$

which proves (ii)'.

It remains to prove (iii). Let $X_{k, i} \subset \partial U$ be such that $X_{k, i} \approx D, \partial X_{k, i}=$ $\partial \hat{Z}_{k, i}$, and $H^{2}\left(X_{k, i}\right) \leq H^{2}\left(\partial U \sim X_{k, i}\right)$. Then from the way we defined $Y_{\alpha}$ from $C_{\alpha}$ (or $Y_{i j}$ from $C_{i j}$ ) we deduce that $X_{k, 1}, X_{k, 2}, \cdots, X_{k, m_{k}}$ are pairwise disjoint and

$$
H^{2}\left(X_{k, j}\right) \leq H^{2}\left(\partial U \sim \bigcup_{i=1}^{m_{k}} X_{k, i}\right)
$$


for each $j=1, \cdots, m_{k}$. Hence by $(2.4)$

$$
\begin{aligned}
H^{2}(\delta \hat{F} \cap U) & =\sum_{k=1}^{n_{2}} \sum_{i=1}^{m_{k}} H^{2}\left(\hat{Z}_{k, i}\right) \leq \sum_{k=1}^{n_{2}} \sum_{i=1}^{m_{k}} H^{2}\left(X_{k, i}\right) \\
& \leq \sum_{k=1}^{n_{2}} c\left(H^{1}\left(\bigcup_{i=1}^{m_{k}} \partial X_{k, i}\right)\right)^{2}=\sum_{k=1}^{n_{2}} c\left(H^{1}\left(\bigcup_{i=1}^{m_{k}} \partial Z_{k, i}\right)\right)^{2} \\
& =\sum_{k=1}^{n_{2}} c\left(H^{1}\left(C_{\alpha_{k-1}}\right)\right)^{2} \leq c\left(H^{1}(\delta \hat{F} \cap \partial U)\right)^{2}
\end{aligned}
$$

This completes the proof of Theorem 1 .

The following lemma is a generalization of the Filigree Lemma in [4]. Here the word "filigree" means (very roughly) a collection of threadlike protrusions from a surface. For example, if $F \in \Phi$ satisfies $H^{2}\left(\delta F \cap B^{3}(p, r)\right)=\varepsilon r^{2}$, where $\varepsilon$ is small, then $\delta F \cap B^{3}(p, r / 2)$ would be classified as filigree.

The following lemma will enable us to "cut off" such sets under appropriate circumstances.

Lemma 3 (Filigree lemma). Let $U_{t}=B^{3}(p, r t), p \in M, r>0,0 \leq t \leq$ 1 , and suppose $U_{t}$ is convex for all $0 \leq t \leq 1$. Suppose also that there is a constant $c<\infty$ such that, whenever $E \subset \partial U_{t}$ is a set homeomorphic to $D$, then $\min \left\{H^{2}(E), H^{2}\left(\partial U_{t} \sim E\right)\right\} \leq c\left(H^{1}(\partial E)\right)^{2}$.

Finally, suppose $F \in \Phi_{0}$ and $\varepsilon>0$ are such that

$$
H^{2}(\delta F) \leq H^{2}(\delta G)+\varepsilon \quad \text { for any } G \in \Phi_{0} .
$$

Then $H^{2}\left(\delta F \cap U_{t}\right) \leq \varepsilon$ whenever $t \leq 1-(2 / r) \sqrt{c} \sqrt{H^{2}\left(\delta F \cap U_{1}\right)}$.

Moreover we can obtain the same result for $F \in \Phi$ with the additional assumption that $M$ is irreducible.

Proof. We will prove the lemma in the case of $\Phi$ only since the proof for $\Phi_{0}$ is basically the same. By Sard's Theorem $\delta F$ intersects $\partial U_{t}$ transversally for almost all $t \in(0,1)$. Then for almost all $t \in(0,1)$ we can apply Theorem 1 , with $U_{t}$ in place of $U$, to give $\hat{F} \in \Phi$ such that

$$
\begin{gathered}
\delta \hat{F} \sim U_{t} \subset \delta F \sim U_{t}, \\
H^{2}\left(\delta \hat{F} \cap U_{t}\right) \leq c\left(H^{1}\left(\delta \hat{F} \cap \partial U_{t}\right)\right)^{2} .
\end{gathered}
$$

By (2.5) we have

$$
H^{2}\left(\delta F \cap U_{t}\right)+H^{2}\left(\delta F \sim U_{t}\right) \leq H^{2}\left(\delta \hat{F} \cap U_{t}\right)+H^{2}\left(\delta \hat{F} \sim U_{t}\right)+\varepsilon,
$$

which together with (2.6) implies

$$
H^{2}\left(\delta F \cap U_{t}\right) \leq H^{2}\left(\delta \hat{F} \cap U_{t}\right)+\varepsilon .
$$


Then (2.7) gives

$$
H^{2}\left(\delta F \cap U_{t}\right) \leq c\left(H^{1}\left(\delta \hat{F} \cap \partial U_{t}\right)\right)^{2}+\varepsilon .
$$

Since (2.6) yields $\delta \hat{F} \cap \partial U_{t} \subset \delta F \cap \partial U_{t}$, we have

$$
H^{2}\left(\delta F \cap U_{t}\right) \leq c\left(H^{1}\left(\delta F \cap \partial U_{t}\right)\right)^{2}+\varepsilon .
$$

We can now suppose $H^{2}\left(\delta F \cap U_{1}\right)>\varepsilon$, otherwise the required conclusion is trivial. Then let $t_{0}=\inf \left\{t: H^{2}\left(\delta F \cap U_{t}\right)>\varepsilon\right\}$ and define $f(t)=$ $H^{2}\left(\delta F \cap U_{t}\right)-\varepsilon, t \in\left[t_{0}, 1\right]$. By the co-area formula we see that (2.8) implies

$$
f(t) \leq \frac{c}{r^{2}}\left(f^{\prime}(t)\right)^{2} \quad \text { a.e. } t \in\left[t_{0}, 1\right] .
$$

Integrating this inequality (using the fact that $f(t)$ is an increasing function of $t$ ), we obtain

$$
\sqrt{f\left(t_{0}\right)} \leq \sqrt{f(1)}-\frac{r\left(1-t_{0}\right)}{2 \sqrt{c}} .
$$

However $f(1)=H^{2}\left(\delta F \cap U_{1}\right)-\varepsilon<H^{2}\left(\delta F \cap U_{1}\right)$; hence we deduce

$$
1-t_{0}<\frac{2}{r} \sqrt{c} \sqrt{H^{2}\left(\delta F \cap Y_{1}\right)} .
$$

That is,

$$
t_{0}>1-\frac{2}{r} \sqrt{c} \sqrt{H^{2}\left(\delta F \cap Y_{1}\right)},
$$

and the required result is proved.

With the above filigree lemma we are now able to get the first regularity result as follows:

First let $\left\{F_{k}\right\}$ be a minimizing sequence in $\Phi_{0}$ (respectively $\Phi$ ), that is, $H^{2}\left(\delta F_{k}\right) \leq H^{2}(\delta G)+\varepsilon_{k}$ for any $G \in \Phi_{0}$ (respectively $\Phi$ ), where $\varepsilon_{k} \rightarrow 0$ as $k \rightarrow \infty$. Then we can apply compactness of varifolds and hence assume, by taking a subsequence if necessary, that there is a 2-varifold $\Delta$ in $M$ such that $\Delta=\lim _{k \rightarrow \infty}\left|\delta F_{k}\right| . \Delta$ is of course area minimizing in $M$ under diffeomorphism because $\mathbf{M}(\Delta) \leq \mathbf{M}\left(\phi_{\#} \Delta\right)$ whenever $\phi$ is a diffeomorphism of $M$. Secondly we want to show that there is a constant $c_{0}>0$ such that whenever $p \in \operatorname{spt}\|\Delta\|$

$$
\Theta^{2}(\|\Delta\|, p) \geq c_{0}
$$

Suppose $p \in \operatorname{spt}\|\Delta\|$ and let $c$ be a constant as defined in the filigree lemma (obviously such $c$ exists and depends only on $M$ and $r$ ). By the filigree lemma we know that if $H^{2}\left(\delta F_{k} \cap B^{3}(p, r)\right) \leq r^{2} /(16 c)$, then $H^{2}\left(\delta F_{k} \cap B^{3}(p, r / 2)\right)<$ $\varepsilon_{k}$. If there is a subsequence $\left\{k^{\prime}\right\} \subset\{k\}$ with $H^{2}\left(\delta F_{k^{\prime}} \cap B^{3}(p, r)\right) \leq r^{2} /(16 c)$, then we would have spt $\|\Delta\| \cap B^{3}(p, r / 2)=\varnothing$, thus contradicting the 
fact that $p \in \operatorname{spt}\|\Delta\|$. Hence for all sufficiently large $k$ we have $H^{2}\left(\delta F_{k} \cap B^{3}(p, r)\right) \geq r^{2} /(16 c)$, from which we deduce

$$
\|\Delta\|\left(B^{3}(p, r)\right) \geq \frac{1}{16 c} r^{2} \text {. }
$$

Thus we obtain (2.9) with $c_{0}=1 /(16 c)$. In particular, $\Delta$ is rectifiable by $[1,5.5]$.

Hence we have proved the following.

Corollary 1 (Rectifiability). Suppose $\left\{F_{k}\right\}$ is a minimizing sequence in $\Phi_{0}$. Then a subsequence of the corresponding varifolds $\left|F_{k}\right|$ converges to a rectifiable 2-varifold $\Delta$ in $M$ which is area minimizing under diffeomorphism and has $M(\Delta)=\inf \left\{H^{2}(\delta G): G \in \Phi_{0}\right\}$. Moreover we can obtain the same result for $F \in \Phi$ assuming that $M$ is irreducible.

Lemma 4 (Monotonicity lemma). Let $\Delta$ be a rectifiable 2-varifold in $M$ which is area minimizing under diffeomorphism of $M$, and $p \in \operatorname{spt}\|\Delta\|$. Let $\rho$ be the injectivity radius of $M$. Then there exists a function $\xi(r)=$ $c r^{m}, c, m>0$, such that the function $g:(0, \rho / 2) \rightarrow \mathbf{R}^{1}$ defined by $g(r)=$ $r^{-2}\|\Delta\|\left(B^{3}(p, r)\right) e^{\xi(r)}$ is monotonically nondecreasing.

Proof. Let $p \star\left(\right.$ spt $\left.\|\Delta\| \cap \partial B^{3}(p, r)\right)$ be the set of all geodesics from $p$ to the points of spt $\|\Delta\| \cap \partial B^{3}(p, r)$. Then there exists a sequence $\left\{\psi_{n}\right\}$ of "shrinking" diffeomorphisms of $M$ such that $\psi_{n}(x)=x$ for all $n$ and $x \in M \sim B^{3}(p, r)$, and

$$
\operatorname{spt}\left\|\lim _{n \rightarrow \infty}\left(\psi_{n \#} \Delta\right)\right\| \cap B^{3}(p, r)=p \notin\left(\operatorname{spt}\|\Delta\| \cap \partial B^{3}(p, r)\right) .
$$

Since $\Delta$ is rectifiable, there is a positive $H^{2}$-measurable function $\theta$ on $N \equiv$ spt $\|\Delta\|$ such that

$$
\Delta(S)=\int_{S \cap N} \theta d H^{2} \text { for any } H^{2} \text {-measurable } S .
$$

Thus, as in $[11, \S 15]$, we adapt the notation $\Delta=\mathbf{v}(N, \theta)$, which is characterized by (2.11). Note that for almost all $r>0, N \cap \partial B^{3}(p, r)$ is rectifiable. Hence a 1 -varifold $\Delta \cap \partial B^{3}(p, r)$ defined by

$$
\Delta \cap \partial B^{3}(p, r)=\mathbf{v}\left(N \cap \partial B^{3}(p, r),\left.\theta\right|_{\partial B^{3}(p, r)}\right)
$$

is a rectifiable varifold for almost all $r$.

Define $p \nVdash\left(\Delta \cap \partial B^{3}(p, r)\right)=\mathbf{v}\left(p \star\left(N \cap \partial B^{3}(p, r), \tilde{\theta}\right)\right)$, where $\tilde{\theta}(\tilde{x})=\theta(x)$ whenever $\tilde{x}$ lies on the geodesic from $p$ to $x \in N \cap \partial B^{3}(p, r)$. Then we can deduce that for almost all $r$,

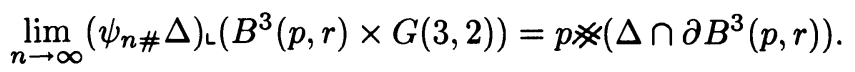

Define $m(r)=\|\Delta\|\left(B^{3}(p, r)\right)$. Since $\Delta$ is area minimizing,

$$
m(r) \leq\left\|\psi_{n \#} \Delta\right\|\left(B^{3}(p, r)\right)
$$


Taking the limit as $n \rightarrow \infty$, we have $\left.m(r) \leq \mathbf{M}(p)\left(\Delta \cap \partial B^{3}(p, r)\right)\right)$.

Now we can find a function $\xi(r)=c r^{m}, c, m>0$, depending on $M$ such that

$$
M\left(p \star\left(\Delta \cap \partial B^{3}(p, r)\right)\right) \leq(1+\xi(r)) \frac{r}{2} \cdot \mathbf{M}\left(\Delta \cap \partial B^{3}(p, r)\right) .
$$

Hence $m(r) \leq \frac{1}{2}(1+\xi(r)) r \cdot \mathbf{M}\left(\Delta \cap \partial B^{3}(p, r)\right)$. Since $m^{\prime}(r)$ exists and $\mathbf{M}\left(\Delta \cap \partial B^{3}(p, r)\right) \leq m^{\prime}(r)$, for almost all $r$, we get

$$
m(r) \leq \frac{1}{2}(1+\xi(r)) r m^{\prime}(r), \quad \text { i.e., } \frac{r}{2} m^{\prime}(r)-m(r)(1-\xi(r)) \geq 0 .
$$

It follows that $r m(r) \frac{d}{d r} \log g(r) \geq 0$ and hence $g^{\prime}(r) \geq 0$.

Corollary 2. Let $\Delta$ be an area minimizing 2-varifold in $M$ obtained as above and $p \in \mathrm{spt}\|\Delta\|$. Then tangent cones to $\Delta$ exist at $p$; the tangent cones are cones in $\mathbf{R}^{3}$ and are area minimizing under diffeomorphism in $\mathbf{R}^{3}$.

Proof. By the Nash embedding theorem we can assume that $M$ is isometrically embedded in $\mathbf{R}^{n}$ for some $n \geq 3$. For convenience of notation we will assume $p=0$. Let $\left\{r_{i}\right\}$ be a sequence of positive radii with $\lim _{i \rightarrow \infty} r_{i}=0$. By Lemma 4 the varifolds

$$
\Delta_{i}=\left(\left(\mu_{1 / r_{i}}\right)_{\#} \Delta\right)_{\llcorner}\left(B^{n}\left(0, r_{i}\right) \times G(n, 2)\right)
$$

all have bounded masses; since their supports also all lie in a bounded region of $\mathbf{R}^{n}$, the varifolds $\left\{\Delta_{i}\right\}$ have a convergent subsequence and a limit varifold $\mu$. By definition $\mu$ is a tangent cone. Now $\mu_{\mathrm{L}}\left(B^{n}(0,1) \times G(n, 2)\right)$ is stationary, since any diffeomorphism of $\mathbf{R}^{n}$ which would decrease the mass of $\mu$ would also decrease uniformly the masses of the varifolds $\Delta_{i}$ for large $i$, contradicting the area minimizing property of $\Delta$. The density ratios of $\mu$ are uniformly bounded away from 0 at each point in spt $\|\mu\|$ since they are uniformly so bounded for $\Delta_{i}$ by (2.9). Therefore by $[1,5.5] \mu_{\llcorner}\left(B^{n}(0,1) \times G(3,2)\right)$ is a rectifiable varifold and the support of $\|\mu\|$ is a rectifiable set $[1,2.8]$. $\mu$ has density at every point in its support at least $(16 c)^{-1}$, since each of the varifolds $\Delta_{i}$ does by $(2.10)$. Therefore we may apply $[1,5.2,6.5]$ to conclude that $\mu$ is in fact a cone. Finally $\mu$ is area minimizing in $\mathbf{R}^{n}$ because any diffeomorphism of $\mathbf{R}^{n}$ which saved mass in $\mu$ would also save mass uniformly in the varifolds $\Delta_{i}$ for large $i$. $\mu$ is obviously area minimizing in $\mathbf{R}^{3}$ too.

\section{Uniformly bounded minimizing sequence}

In order to obtain the desired existence and regularity result for both first and second problems, it is desirable to replace the given minimizing sequence of fundamental domains in $\Phi_{0}$ or $\Phi$ by another minimizing sequence (with the same limit) of uniformly bounded ones in $\Phi_{0}$ or $\Phi$ without filigrees. 
In this section we use a cutting and pasting argument extensively. In this process however we have to be very careful not to change the topology of fundamental domains in the case of the second problem. The following lemma allows us to cut and paste fundamental domains without changing their topology.

Lemma 5 (Pasting lemma). (1) Let $F$ be an adequate fundamental domain in $\Phi$. If there exists a face $X$ of $F$ which is multiply connected, then $M$ is not irreducible.

(2) Let $M$ be irreducible. In case $F$ is an inadequate fundamental domain in $\Phi$, there may exist a face $X$ of $F$ which is multiply connected. But then $X$ can be replaced by a face homeomorphic to $D$ in the following sense: For any nonnull-homotopic Jordan curve $C$ on $X$, there exist $\hat{F} \in \Phi$ and $U \subset M$ with $U \approx B^{\circ}$ such that $\partial U \cap \delta F=p(C), \delta \hat{F} \sim U=\delta F \sim U$, and $\delta \hat{F} \cap U \subset p(E)$, where $E$ is a face of $\hat{F}$ homeomorphic to $D$.

Proof. (1) By assumption there exists a face $Y \neq X$ of $F$ such that $X=$ $g(Y)$ for some translation $g$. Let $S_{1}$ and $S_{2}$ be such that $S_{1}, S_{2} \subset \bar{F}, S_{1}$, $S_{2} \approx \bar{D}, \partial S_{1}=S_{1} \cap \partial F \subset X, \partial S_{2}=S_{2} \cap \partial F \subset Y, p\left(\partial S_{1}\right)=p\left(\partial S_{2}\right), \partial S_{1}$ and $\partial S_{2}$ are not null-homotopic in $X$ and $Y$ respectively, and $S_{1} \cap S_{2}=\varnothing$. Then $p\left(S_{1} \cap S_{2}\right)$ is a closed surface in $M$ homeomorphic to a sphere. Suppose $M$ is irreducible. Define $S=p\left(S_{1} \cup S_{2}\right)$ and let $K$ be the component of $M \sim S$ which is homeomorphic to $B^{\circ}$. Then $S_{1} \cup g\left(S_{2}\right)$ is also a closed surface in $\tilde{M}$ homeomorphic to a sphere. Let $\hat{K}$ be the component of $\tilde{K}$ with $\partial \hat{K}=S_{1} \cup g\left(S_{2}\right)$. Now since by hypothesis $\hat{K} \sim(F \cup g(F))$ is nonempty, we can find a translation $h$ such that $h(F) \subset \hat{K} \sim(F \cup g(F))$. We then have

$$
H^{3}(M)=H^{3}(h(F))<H^{3}(\hat{K})<H^{3}(M),
$$

which is a contradiction. Thus $M$ is not irreducible.

(2) As in the proof of (1) we can find $Y, g, S_{1}, S_{2}$, and $K$ satisfying the same properties. Here we assume $\partial S_{1}=C$. Since $S_{1} \cup S_{2}$ divides $F$ into three components, $\tilde{K} \cap F$ is either connected or $\tilde{K} \cap F$ consists of two components. Suppose $\tilde{K} \cap F$ is connected and let $L_{1}, L_{2}$ be the remaining components of $F \sim\left(S_{1} \cup S_{2}\right)$, i.e., $F \sim\left(S_{1} \cup S_{2}\right)=(\tilde{K} \cap F) \cup L_{1} \cup L_{2}$. Clearly $L_{1}, L_{2} \approx B^{\circ}$. Define $Z=\partial F \cap \tilde{K}$. Then we have $H^{2}(Z)>0$ and $p(Z) \subset K$. We thus note that since $K, p\left(L_{1}\right), p\left(L_{2}\right)$ are all homeomorphic to $B^{\circ}, K \cup p\left(L_{1}\right) \cup p\left(L_{2}\right) \cup p\left(S_{1}^{\circ}\right) \cup p\left(S_{2}^{\circ}\right)$ is also homeomorphic to $B^{\circ}$, where $S_{i}^{\circ}=S_{i} \sim \partial S_{i}$. Note also that

$$
M \sim\left(K \cup p\left(L_{1}\right) \cup p\left(L_{2}\right) \cup p\left(S_{1}^{\circ}\right) \cup p\left(S_{2}^{\circ}\right)\right)=\delta F \sim p(Z),
$$

since $p(Z) \subset K$. Hence $\delta F \sim p(Z)$ is a spine of $M$ which is a proper subset of $\delta F$. Therefore $F$ is reducible. Since this contradicts the hypothesis that $F$ is in $\Phi, \tilde{K} \cap F$ must consist of two components. 
Define again $Z=\partial F \cap \tilde{K}$ and $L=F \sim\left(S_{1} \cup S_{2} \cup \tilde{K}\right)$. Then both $K \cup p(L) \cup p\left(S_{1}^{\circ}\right)$ and $K \cup p(L) \cup p\left(S_{2}^{\circ}\right)$ are homeomorphic to $B^{\circ}$. Let $\hat{F}_{i} \in \Phi$ be such that $p\left(\hat{F}_{i}\right)=K \cup p(L) \cup p\left(S_{i}^{\circ}\right), i=1,2$. Then we see that $\delta \hat{F}_{i}=M \sim$ $\left(K \cup p(L) \cup p\left(S_{i}^{\circ}\right)\right), \delta \hat{F}_{i} \sim \bar{K}=\delta F \sim \bar{K}, \delta \hat{F}_{1} \cap \bar{K}=p\left(S_{2}\right)$, and $\delta \hat{F}_{2} \cap \bar{K}=p\left(S_{1}\right)$. Now we can find $U \supset K$ with $U \approx B^{\circ}$ and $\partial U \cap \delta \hat{F}_{i}=p(C)$. Finally let $\hat{F}$ be either $\hat{F}_{1}$ or $\hat{F}_{2}$. Then $U$ and $\hat{F}$ satisfy the desired properties.

Remark 1. (1) One can conclude from the above lemma that $\Phi$ is "closed" under cutting and pasting provided $M$ is irreducible: Any $S \subset \bar{F}$ with $S \approx \bar{D}$ and $S \cap \partial F=\partial S$ cuts $F$ into two components, $V_{1}$ and $V_{2}$. Suppose $E_{1}, E_{2}$ are faces of $F$ with $E_{i} \subset \partial V_{i}, i=1,2$, and $E_{1}=g\left(E_{2}\right)$ for some translation $g$. We then translate $V_{2}$ via $g$ and paste $g\left(V_{2}\right)$ to $V_{1}$ along $E_{1}$. The resulting fundamental domain $\hat{F} \equiv V_{1} \cup g\left(V_{2}\right) \cup E_{1}$ is homeomorphic to $B^{\circ}$ by (1) of the above lemma in case $F$ is adequate, and by (2) in case $F$ is inadequate. Hence $\hat{F} \in \Phi_{1}$. Since $\hat{F}$ may be reducible, we may have to eliminate the appropriate face (redundant face) of $\hat{F}$ to obtain a fundamental domain which is not reducible. Figure 6 illustrates two pathological cases; an inadequate fundamental domain with an annular face and a reducible fundamental domain with a redundant face (review terminology (1)).

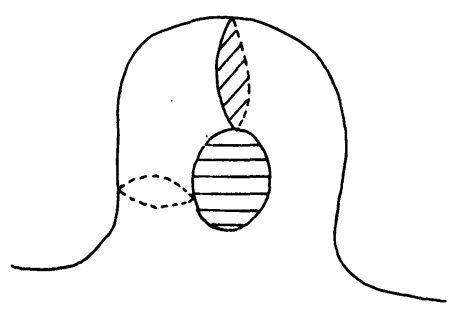

(a)

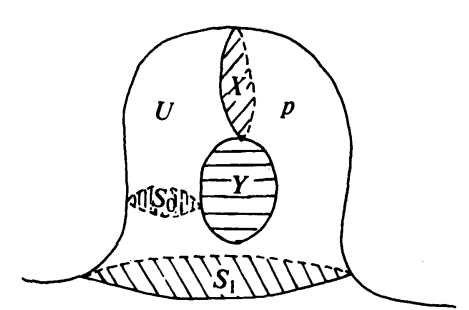

(b)

FiguRE 6 (in $M$ )

Figures 6(a) and 6(b) are basically the same pictures of a part of $\delta F$ in $M$ which is topologically a punctured torus with two disks $X$ and $Y$ added. The two shaded disks, $X$ and $Y$, are faces of $\delta F$. $F$ is inadequate since $\bar{F}$ has two solid handles corresponding to the interior and the exterior of the (punctured) torus. $\partial X \cap \partial Y(\equiv\{p\})$ is a multiple point of $\delta F$, and $\partial X \cup \partial Y \sim\{p\}$ is the union of two multiple curves of $\delta F$. The face of $\delta F$ on which the dotted circle (= $\partial S_{0}$ ) lies is homeomorphic to an annulus. In Figure (b) $S_{0}$ cuts $M \sim \delta F$ into two components, $U$ and $M \sim(\delta F \cup U)$. So we paste $U$ to $M \sim(\delta F \cup U)$ along $\partial U \sim\left(X \cup S_{0}\right)$ to get a fundamental domain $\hat{F}$. But $\hat{F}$ is reducible 
since the face $Y$ of $\delta \hat{F}$ is redundant (i.e., $\delta \hat{F} \sim Y$ is still a spine of $M$ ). The fundamental domain $F^{\prime}$ with $\delta F^{\prime}=\delta \hat{F} \sim Y$ will then be not reducible, or $F^{\prime} \in \Phi$. On the other hand, $S_{1}$ cuts $M \sim \delta F$ into $W \supset U$ and $M \sim(\delta F \cup W)$. Although $W$ has an annular face $\partial W \sim\left(\bar{X} \cup \bar{Y} \cup \bar{S}_{1}\right)$, we can paste $W$ to $M \sim(\delta F \cup W)$ along the annular face and eliminate (i.e., fill up) $\bar{X}$ and $\bar{Y}$ to obtain $\hat{F} \in \Phi$ with $\delta \hat{F}=[\delta F \sim(\partial W \cup Y)] \cup S_{1}$. This illustrates Lemma 5(2).

(2) Unlike $\Phi, \Phi_{2}$ is not closed under cutting and pasting: Let $F$ be a fundamental domain of $M$ in $\Phi_{2}$. Suppose $E \subset F, E \approx D$, and $\partial E \subset \partial F$. Then $E$ cuts $F$ in two components $U$ and $V$. Suppose $X, Y, Z, W$ are faces of $F$ with $X, Y \subset \partial V$ and $Z, W \subset \partial U$ such that $Z=\tau(X)$ and $W=\tau(Y)$ for some translation $\tau$. Then $U \cup \tau(V) \cup Z$ is a fundamental domain in $\Phi$ but not in $\Phi_{2}$ since the closure of $U \cup \tau(V) \cup Z$ is topologically a solid torus, as is illustrated by Figure 7 .
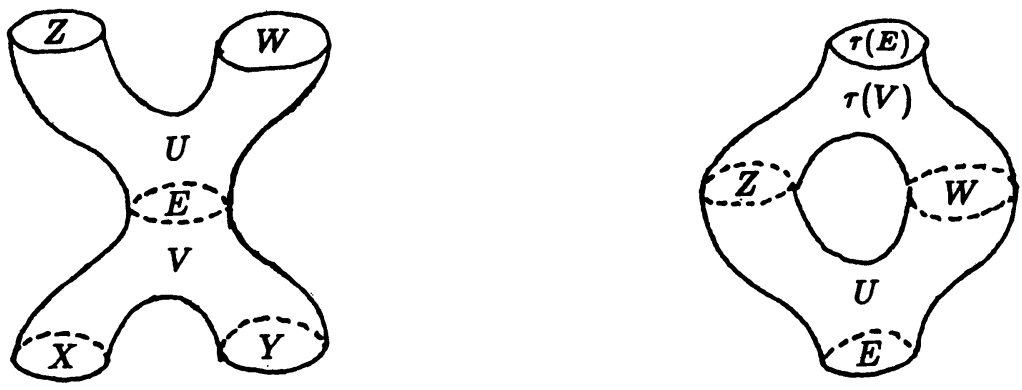

FIGURE 7 (in $M$ )

Theorem 2 (Boundedness theorem). Suppose $\left\{F_{k}\right\}$ is a sequence in $\Phi_{0}$ such that $H^{2}\left(\delta F_{k}\right) \leq H^{2}(\delta G)+\varepsilon_{k}$ for any $G \in \Phi_{0}$, where $\varepsilon_{k} \rightarrow 0$ as $k \rightarrow \infty$. Then $\left\{F_{k}\right\}$ can be replaced by another minimizing sequence $\left\{F_{k}^{4}\right\}$ in $\Phi_{0}$ which is uniformly bounded in $\tilde{M}$. Moreover we can obtain the same result for a minimizing sequence in $\Phi$ if $M$ is irreducible.

Proof of Theorem 2. We will prove this theorem in the case of $\Phi$ only, and then we will be able to see that the same argument is valid for the case of $\Phi_{0}$ since the cutting and pasting method in $\Phi_{0}$ (Lemma 2(1)) is much simpler than that in $\Phi$ (Lemma 5, Remark 1(1)).

In this proof we concern ourselves mainly with homothetic expansion and the cutting and pasting argument. Therefore we prove the theorem first assuming that $M$ is locally isometrically in $\mathbf{R}^{3}$, and later we shall see that the theorem in general can be proved similarly by using the exponential map.

Suppose $p=0$ for convenience. We write

$$
K_{\rho, \sigma}=\left\{x \in B^{3}(0, \rho): \operatorname{dist}(x, \operatorname{spt}\|\mu\|) \leq \sigma\right\} .
$$


By definition of $\mu$, we know there is a sequence $\left\{r_{k}\right\} \rightarrow \infty$ such that $\mu_{r_{k} \#} \Delta \rightarrow$ $\mu$ as $k \rightarrow \infty$. By $(2.10)$ it is then clear that for any $\sigma_{0} \in(0,1)$ we can find $r$ such that

$$
B^{3}(0,1) \cap \operatorname{spt}\left\|\mu_{r \#} \Delta\right\| \subset K_{1, \sigma_{0} / 2} .
$$

Define $J_{\rho, \sigma}=U^{3}(0, \rho) \sim K_{\rho, \sigma}$ and $L_{\delta}=\left\{x: x \in J_{1, \sigma_{0} / 2}\right.$, dist $\left(x, \partial J_{1, \sigma_{0} / 2}\right)=$ $\delta\}$ for $\delta>0$. Then from (3.1) and the co-area formula it follows that for almost all $\sigma \in\left(\sigma_{0} / 2,1 / 2\right), H^{1}\left(\mu_{r}\left(\delta F_{k}\right) \cap L_{\sigma-\sigma_{0} / 2}\right) \rightarrow 0$ as $k \rightarrow \infty$. Thus for any given $\eta>0$ we can assert that, for sufficiently large $k$, there is a $\sigma_{k} \in\left(3 / 4 \sigma_{0}, \sigma_{0}\right)$ such that

$$
H^{1}\left(\mu_{r}\left(\delta F_{k}\right) \cap L_{\sigma_{k}-\sigma_{0} / 2}\right)<\eta .
$$

We can also arrange, by Sard's theorem, that $\mu_{r}\left(\delta F_{k}\right)$ intersects $L_{\sigma_{k}-\sigma_{0} / 2}$ transversally. Now we claim the following lemma.

Lemma 6. For any $\sigma_{0} \in(0,1)$ there is an $r>0$ such that every filigree, or spike, of $\mu_{r}\left(\delta F_{k}\right)$ passing through $J_{1-\sigma_{k}+\sigma_{0} / 2, \sigma_{k}}$ can be eliminated to make a new minimizing sequence $\left\{\delta F_{k}^{2}\right\}$ with the same varifold limit $\Delta$. (That is, every filigree of $\mu_{r}\left(\delta F_{k}\right)$ passing through $J_{1-\sigma_{k}+\sigma_{0} / 2, \sigma_{k}}$ can be cut off without changing varifold limit $\Delta$.)

Proof of Lemma 6. Choose $r$ as in (3.1). Note that

$$
L_{\sigma_{k}-\sigma_{0} / 2}=\partial\left(J_{1-\sigma_{k}+\sigma_{0} / 2}, \sigma_{k}\right) \text {. }
$$

Hence we apply Theorem 1 repeatedly with $\mu_{r}\left(\delta F_{k}\right)$ in place of $\delta F, \mu_{r}\left(F_{k}\right)$ in place of $F$, and each component of $J_{1-\sigma_{k}+\sigma_{0} / 2, \sigma_{k}}$ in place of $U$. Then we get $F_{k}^{1} \in \Phi$ and $Z_{1}, Z_{2}, \cdots, Z_{n_{k}}$ which are homeomorphic to $D$ such that

$$
\mu_{r}\left(\delta F_{k}^{1}\right) \cap J_{1-\sigma_{k}+\sigma_{0} / 2, \sigma_{k}}=\bigcup_{i=1}^{n_{k}} Z_{i}
$$

$$
\mu_{r}\left(\delta F_{k}^{1}\right) \sim J_{1-\sigma_{k}+\sigma_{0} / 2, \sigma_{k}} \subset \mu_{r}\left(\delta F_{k}\right) \sim J_{1-\sigma_{k}+\sigma_{0} / 2, \sigma_{k}}
$$

Hence, as in the proof of Theorem 1 , we have $\left\{C_{\alpha}\right\}$ with

$$
\bigcup_{\alpha} C_{\alpha}=\bigcup_{i=1}^{n_{k}} \partial Z_{i}=\mu_{r}\left(\delta F_{k}^{1}\right) \cap L_{\sigma_{k}-\sigma_{0} / 2} \subset \mu_{r}\left(\delta F_{k}\right) \cap L_{\sigma_{k}-\sigma_{0} / 2}
$$

and the corresponding $\left\{Y_{\alpha}\right\}$ with $Y_{\alpha} \subset L_{\sigma_{k}-\sigma_{0} / 2}$ and $Y_{\alpha} \approx D$. The next step involves a slight perturbation of $\bigcup_{i=1}^{n_{k}} Z_{i}$, holding the set $\mu_{r}\left(\delta F_{k}^{1}\right) \sim$ $J_{1-\sigma_{k}+\sigma_{0} / 2, \sigma_{k}}$ fixed, and taking points of $\bigcup_{i=1}^{n_{k}} Z_{i}$ into $\mu_{r}(M) \sim \bar{J}_{1-\sigma_{k}+\sigma_{0} / 2, \sigma_{k}}$ in such a way that each $Z_{i}$ is taken closely to some $Y_{\alpha}$. In this way we can 
obtain $F_{k}^{2} \in \Phi$ such that

$$
H^{2}\left(\mu_{r}\left(\delta F_{k}^{2}\right)\right) \leq H^{2}\left(\mu_{r}\left(\delta F_{k}\right)\right)+H^{2}\left(\bigcup_{\alpha} Y_{\alpha}\right)+\delta
$$

for any preassigned $\delta>0$. Since $\bigcup_{\alpha} \partial Y_{\alpha} \subset \bigcup_{\alpha} C_{\alpha}=\mu_{r}\left(\delta F_{k}^{1}\right) \cap L_{\sigma_{k}-\sigma_{0} / 2}$ we have by (3.2) and (3.3),

$$
H^{2}\left(\bigcup_{\alpha} Y_{\alpha}\right) \leq \sum_{\alpha} a_{p}\left(H^{1}\left(\partial Y_{\alpha}\right)\right)^{2} \leq a_{p} \eta^{2}
$$

where $a_{p}$ depends on the point $p$ and is finite because, for fixed $\sigma_{0}>0$, $L_{\sigma_{k}-\sigma_{0} / 2}$ cannot have an arbitrarily sharp vertex. Thus, taking $\delta$ and $\eta$ arbitrarily small, we deduce from (3.6) and (3.7) that

$$
\lim _{k \rightarrow \infty} H^{2}\left(\mu_{r}\left(\delta F_{k}^{2}\right)\right) \leq \lim _{k \rightarrow \infty} H^{2}\left(\mu_{r}\left(\delta F_{k}\right)\right) .
$$

It follows from (3.5) that $\lim _{k \rightarrow \infty}\left|\delta F_{k}^{2}\right|=\lim _{k \rightarrow \infty}\left|\delta F_{k}\right|$. This proves Lemma 6.

Now choose $\rho_{k}$ with $7 / 8 \leq \rho_{k} \leq 1-\sigma_{k}$ (assuming $0<\sigma_{0}<1 / 8$ ) such that $\mu_{r}\left(\delta F_{k}^{2}\right)$ is transversal to $\partial B^{3}\left(0, \rho_{k}\right)$. Note that by $(3.4)$

$$
\mu_{r}\left(\delta F_{k}^{2}\right) \cap \partial B^{3}\left(0, \rho_{k}\right)=\mu_{r}\left(\delta F_{k}^{2}\right) \cap \partial B^{3}\left(0, \rho_{k}\right) \cap K_{1, \sigma_{k}} .
$$

Hence $\mu_{r}\left(\delta F_{k}^{2}\right) \cap \partial B^{3}\left(0, \rho_{k}\right)=\bigcup_{i=1}^{l_{k}} C_{k}^{i}$, where each $C_{k}^{i}$ is a compound Jordan curve on $\partial B^{3}\left(0, \rho_{k}\right)$. Let $Y_{k}^{i}$ be the open disk in $\partial B^{3}\left(0, \rho_{k}\right)$ corresponding to $C_{k}^{i}$ as defined in the proof of Theorem 1. Assuming $\sigma_{0}$ is taken small enough, we can deduce that if $C_{k}^{i}$ is null-homotopic in $\partial B^{3}\left(0, \rho_{k}\right) \cap K_{1, \sigma_{k}}$, then

$$
Y_{k}^{i} \subset \partial B^{3}\left(0, \rho_{k}\right) \cap K_{1, \sigma_{k}} .
$$

Let $\left\{Q_{k}^{1}, \cdots, Q_{k}^{a_{k}}\right\}$ be the set of all components of $\mu_{r}\left(\delta F_{k}^{2}\right) \cap B^{3}\left(0, \rho_{k}\right)$. Then each component $Q_{k}^{j}$ falls on one of the following two cases:

Case I. All components of $Q_{k}^{j} \cap \partial B^{3}\left(0, \rho_{k}\right)$ are null-homotopic in $\partial B^{3}\left(0, \rho_{k}\right)$ $\cap K_{1, \sigma_{k}}$.

Case II. A component of $Q_{k}^{j} \cap \partial B^{3}\left(0, \rho_{k}\right)$ is not null-homotopic.

Now we claim the following.

Lemma 7. Every component of $\mu_{r}\left(\delta F_{k}^{2}\right) \cap B^{3}\left(0, \rho_{k}\right)$ of Case I can be discarded to produce another minimizing sequence $\left\{\delta F_{k}^{3}\right\}$ with the same varifold 
limit $\Delta$. (That is, every filigree of $\mu_{r}\left(\delta F_{k}^{2}\right)$ passing through $K_{\rho_{k}, \sigma_{k}}$ can be cut off without changing varifold limit.)

Proof of Lemma 7. Notice that if $C_{k}^{i}$ is null-homotopic in $\partial B^{3}\left(0, \rho_{k}\right) \cap$ $K_{1, \sigma_{k}}$ and $C_{k}^{j}$ is not null-homotopic, then $Y_{k}^{j}$ cannot be a subset of $Y_{k}^{i}$. Moreover if $C_{k}^{i} \subset Q_{k}^{\bar{i}} \cap \partial B^{3}\left(0, \rho_{k}\right)$ and $C_{k}^{j} \subset Q_{k}^{\bar{j}} \cap \partial B^{3}\left(0, \rho_{k}\right)$, where $Q_{k}^{\bar{i}}$ is the component of Case I and $Q_{k}^{\bar{j}}$ is that of Case II, then $Y_{k}^{j}$ cannot be a subset of $Y_{k}^{i}$. Therefore we can find a suitable order $C_{k}^{1}<C_{k}^{2}<\cdots<C_{k}^{l_{k}}$, and renumber $\left\{Q_{k}^{j}\right\}_{1 \leq j \leq a_{k}}$ in such a way that

(i) $\bigcup_{i=1}^{L_{k}} C_{k}^{i}=\bigcup_{j=1}^{A_{k}} Q_{k}^{j} \cap \partial B^{3}\left(0, \rho_{k}\right)$, where $\left\{Q_{k}^{1}, \cdots, Q_{k}^{A_{k}}\right\}$ is the set of all $Q_{k}^{j}$ 's of Case I;

(ii) $\bigcup_{i=L_{k}+1}^{l_{k}} C_{k}^{i}=\bigcup_{j=A_{k}+1}^{a_{k}} Q_{k}^{j} \cap \partial B^{3}\left(0, \rho_{k}\right)$, where $\left\{Q_{k}^{A_{k}+1}, \cdots, Q_{k}^{a_{k}}\right\}$ is the set of all $Q_{k}^{j}$ 's of Case II.

Similarly if we assume $\mu_{r}\left(\delta F_{k}^{2}\right)$ is transversal to $\partial B^{3}(0, t)$ and let

$$
\mu_{r}\left(\delta F_{k}^{2}\right) \cap \partial B^{3}(0, t)=\bigcup_{i=1}^{l_{k}^{t}} C_{k}^{i, t}, \quad 0<t \leq \rho_{k},
$$

then we can find a suitable order $C_{k}^{1, t}<\cdots<C_{k}^{l_{k}^{t}, t}$ such that

$$
\bigcup_{i=1}^{L_{k}^{t}} C_{k}^{i, t}=\bigcup_{j=1}^{A_{k}} Q_{k}^{j} \cap \partial B^{3}(0, t), \quad \bigcup_{i=L_{k}^{t}+1}^{l_{k}^{t}} C_{k}^{i, t}=\bigcup_{j=A_{k}+1}^{a_{k}} Q_{k}^{j} \cap \partial B^{3}(0, t) .
$$

Obviously $C_{k}^{i, \rho_{k}}=C_{k}^{i}, l_{k}^{\rho_{k}}=l_{k}$, and $L_{k}^{\rho_{k}}=L_{k}$.

We then apply the process used in the proof of Theorem 1 (with $\mu_{r}\left(\delta F_{k}^{2}\right)$ in place of $\delta F$ and $U^{3}(0, t)$ in place of $U$, and so on) only for $\left\{C_{k}^{1, t}, \cdots, C_{k}^{L_{k}^{t}, t}\right\}$ (i.e., cutting off the components $Q_{k}^{j}$ of Case I only) until we get $\mu_{r}\left(\delta F_{k}^{2}\right)$, $\mu_{r}\left(\delta F_{k, 1}^{2, t}\right), \cdots, \mu_{r}\left(\delta F_{k, L(t)}^{2, t}\right)$, and $U^{3}(0, t) \supset U_{k, 1}^{t} \supset \cdots \supset U_{k, L(t)}^{t}$ such that $L(t) \leq L_{k}^{t}$

$$
\begin{gathered}
\mu_{r}\left(\delta F_{k, L(t)}^{2, t}\right) \sim U^{3}(0, t) \subset \mu_{r}\left(\delta F_{k}^{2}\right) \sim U^{3}(0, t), \\
\mu_{r}\left(\delta F_{k, L(t)}^{2, t}\right) \cap U_{k, L(t)}^{t}=\bigcup_{j=A_{k}+1}^{a_{k}} Q_{k}^{j} \cap U^{3}(0, t), \\
\mu_{r}\left(\delta F_{k, L(t)}^{2, t}\right) \cap\left(U^{3}(0, t) \sim U_{k, L(t)}^{t}\right)=\bigcup_{\alpha=1}^{\bar{L}(t)} \bigcup_{\beta=1}^{m_{\alpha}} Z_{k, \beta}^{\alpha, t},
\end{gathered}
$$

where $\bar{L}(t) \leq L(t), Z_{k, \beta}^{\alpha, t}$ is an area minimizing surface homeomorphic to $D$, and

$$
\bigcup_{\beta=1}^{m_{a}} \partial Z_{k, \beta}^{\alpha, t}=C_{k}^{n_{\alpha}, t} \quad \text { for some } 1 \leq n_{\alpha} \leq L_{k}^{t}
$$


By Lemma 6 we have

$$
H^{2}\left(\delta F_{k}^{2}\right) \leq H^{2}(\delta G)+\varepsilon_{k}, \quad \text { for any } G \in \Phi,
$$

where $\varepsilon_{k} \rightarrow 0$ as $k \rightarrow \infty$. Thus

$$
\begin{aligned}
& H^{2}\left(\mu_{r}\left(\delta F_{k}^{2}\right) \cap B^{3}(0, t)\right)+H^{2}\left(\mu_{r}\left(\delta F_{k}^{2}\right) \sim B^{3}(0, t)\right) \\
& \quad \leq H^{2}\left(\mu_{r}\left(\delta F_{k, L(t)}^{2, t}\right) \cap B^{3}(0, t)\right)+H^{2}\left(\mu_{r}\left(\delta F_{k, L(t)}^{2, t}\right) \sim B^{3}(0, t)\right)+r^{2} \varepsilon_{k},
\end{aligned}
$$

which together with (3.9) implies

$$
H^{2}\left(\mu_{r}\left(\delta F_{k}^{2}\right) \cap B^{3}(0, t)\right)=H^{2}\left(\mu_{r}\left(\delta F_{k, L(t)}^{2, t}\right) \cap B^{3}(0, t)\right)+r^{2} \varepsilon_{k} .
$$

Hence by (3.10) and (3.11) we have

$$
\begin{aligned}
\sum_{j=1}^{A_{k}} H^{2}\left(Q_{k}^{j} \cap B^{3}(0, t)\right)+\sum_{j=A_{k}+1}^{a_{k}} H^{2}\left(Q_{k}^{j} \cap B^{3}(0, t)\right) \\
\quad \leq \sum_{\alpha=1}^{\bar{L}(t)} \sum_{\beta=1}^{m_{\alpha}} H^{2}\left(Z_{k, \beta}^{\alpha, t}\right)+\sum_{j=A_{k}+1}^{a_{k}} H^{2}\left(Q_{k}^{j} \cap B^{3}(0, t)\right)+r^{2} \varepsilon_{k}
\end{aligned}
$$

or

$$
\sum_{j=1}^{A_{k}} H^{2}\left(Q_{k}^{j} \cap B^{3}(0, t)\right) \leq \sum_{\alpha=1}^{\bar{L}(t)} \sum_{\beta=1}^{m_{\alpha}} H^{2}\left(Z_{k, \beta}^{\alpha, t}\right)+r^{2} \varepsilon_{k} .
$$

Next we claim that $\left|\bigcup_{j=i}^{A_{k}} Q_{k}^{j} \cap B^{3}(0,3 / 4)\right|$ vanishes as $k \rightarrow \infty$. Note first that there are connected subsets $R_{k}^{1}, \cdots, R_{k}^{\bar{L}\left(\rho_{k}\right)}$ of $\mu_{r}\left(\delta F_{k}^{2}\right)$ such that

$$
\bigcup_{\alpha=1}^{\bar{L}\left(\rho_{k}\right)} R_{k}^{\alpha} \cap B^{3}\left(0, \rho_{k}\right)=\bigcup_{j=1}^{A_{k}} Q_{k}^{j} \cap B^{3}\left(0, \rho_{k}\right),
$$

and $\partial R_{k}^{\alpha}=\bigcup_{\beta=1}^{m_{a}} \partial Z_{k, \beta}^{\alpha, \rho_{k}}\left(=C_{k}^{n_{\alpha}}\right)$.

To justify the above claim we show that there exist nonnegative numbers $\varepsilon_{k}^{1}, \cdots, \varepsilon_{k}^{\bar{L}\left(\rho_{k}\right)}$ with

$$
\sum_{\alpha=1}^{\bar{L}\left(\rho_{k}\right)} \varepsilon_{k}^{\alpha} \leq r^{2} \varepsilon_{k}
$$

such that

$$
H^{2}\left(R_{k}^{\alpha} \cap V\right) \leq H^{2}(E)+\varepsilon_{k}^{\alpha}
$$

whenever $V \subset M$ and $E \subset \partial V$ are such that

$V \approx B^{\circ}, \partial V \cap R_{k}^{\alpha}$ is a nonempty compound Jordan curve in $\bar{E}$,

$V \cap Y_{k}^{n_{\alpha}}=\varnothing$, and $\left(\mu_{r}\left(\delta F_{k}^{2}\right) \sim\left(R_{k}^{\alpha} \cap V\right)\right) \cup E=\mu_{r}(\delta G)$

for some $G \in \Phi$. 
Suppose (3.16) fails; then we must have

$$
\sup \left\{H^{2}\left(R_{k}^{\alpha} \cap V\right)-H^{2}(E)\right\}=\theta_{k}^{\alpha},
$$

where $\alpha=1, \cdots, \bar{L}\left(\rho_{k}\right), \sum_{\alpha=1}^{\bar{L}\left(\rho_{k}\right)} \theta_{k}^{\alpha}>r^{2} \varepsilon_{k}$, and the supremum is taken over all $V$ and $E$ satisfying (3.17). Choose $V_{1}, \cdots, V_{\bar{L}\left(\rho_{k}\right)}, E_{1}, \cdots, E_{\bar{L}\left(\rho_{k}\right)}$ with

$$
H^{2}\left(R_{k}^{\alpha} \cap V_{\alpha}\right)-H^{2}\left(E_{\alpha}\right)>\bar{\theta}_{k}^{\alpha}
$$

$$
\sum_{\alpha=1}^{\bar{L}\left(\rho_{k}\right)} \bar{\theta}_{k}^{\alpha}>r^{2} \varepsilon_{k}
$$

and define $R_{k}^{1, \alpha}=\left(R_{k}^{\alpha} \sim V_{\alpha}\right) \cup E_{\alpha}$. Then (3.18) implies

$$
H^{2}\left(R_{k}^{\alpha}\right)-H^{2}\left(R_{k}^{1, \alpha}\right)>\bar{\theta}_{k}^{\alpha} \text {. }
$$

Now we assert $\left\{R_{k}^{1, \alpha}\right\}_{1 \leq \alpha \leq \bar{L}\left(\rho_{k}\right)}$ can be replaced by a pairwise disjoint family satisfying (3.20). The proof of this assertion is by induction on $\alpha$. The result is trivial if $\bar{L}\left(\rho_{k}\right)=1$. Hence take $\bar{L}\left(\rho_{k}\right) \geq 2$ and assume that $\left\{R_{k}^{1, \alpha}\right\}_{1 \leq \alpha \leq \bar{L}\left(\rho_{k}\right)-1}$ is pairwise disjoint. Let $R_{k}^{2, \alpha}$ be the subset of $R_{k}^{1, \alpha}$ homeomorphic to $\bar{D}$ with $\partial R_{k}^{2, \alpha}=\partial Y_{k}^{n_{\alpha}}$. Applying Lemma 2 of [9] to $\left\{R_{k}^{2, \alpha}\right\}_{1 \leq \alpha \leq \bar{L}\left(\rho_{k}\right)}$ we get a pairwise disjoint family $\left\{R_{k}^{3, \alpha}\right\}_{1 \leq \alpha \leq \bar{L}\left(\rho_{k}\right)}$ with $\partial R_{k}^{3, \alpha}=\partial R_{k}^{2, \alpha}$ and $H^{2}\left(R_{k}^{3, \alpha}\right) \leq H^{2}\left(R_{k}^{2, \alpha}\right)$. It is obvious from the proof of this lemma that $\left\{R_{k}^{3, \alpha}\right\}_{1 \leq \alpha \leq \bar{L}\left(\rho_{k}\right)}$ gives rise to $\left\{R_{k}^{4, \alpha}\right\}_{1 \leq \alpha \leq \bar{L}\left(\rho_{k}\right)}$ which is pairwise disjoint and satisfies

$$
H^{2}\left(R_{k}^{\alpha}\right)-H^{2}\left(R_{k}^{4, \alpha}\right)>\bar{\theta}_{k}^{\alpha}
$$

thereby proving our assertion.

Replacing $\left\{R_{k}^{\alpha}\right\}_{1 \leq \alpha \leq \bar{L}\left(\rho_{k}\right)}$ by $\left\{R_{k}^{4, \alpha}\right\}_{1 \leq \alpha \leq \bar{L}\left(\rho_{k}\right)}$, we obtain a new fundamental domain $G \in \bar{\Phi}$; that is,

$$
\left(\mu_{r}\left(\delta F_{k}^{2}\right) \sim\left(\bigcup_{\alpha=1}^{\bar{L}\left(\rho_{k}\right)} R_{k}^{\alpha}\right)\right) \cup\left(\bigcup_{\alpha=1}^{\bar{L}\left(\rho_{k}\right)} R_{k}^{4, \alpha}\right)=\mu_{r}(\delta G)
$$

for some $G \in \Phi$. Therefore

$$
H^{2}\left(\mu_{r}\left(\delta F_{k}^{2}\right)\right)-H^{2}\left(\mu_{r}(\delta G)\right)=\sum_{\alpha=1}^{\bar{L}\left(\rho_{k}\right)}\left[H^{2}\left(R_{k}^{\alpha}\right)-H^{2}\left(R_{k}^{4, \alpha}\right)\right] .
$$

Hence (3.19) and (3.21) give $H^{2}\left(\mu_{r}\left(\delta F_{k}^{2}\right)\right)-H^{2}\left(\mu_{r}(\delta G)\right)>r^{2} \varepsilon_{k}$, contradicting (3.12). Thus (3.16) follows. 
Now let us justify the above mentioned claim. If we define $f_{k}^{\alpha}(t)=$ $H^{2}\left(R_{k}^{\alpha} \cap B^{3}(0, t)\right)-\varepsilon_{k}^{\alpha}, t \in\left[t_{k}^{\alpha}, \rho_{k}\right], t_{k}^{\alpha} \equiv \inf \left\{t: f_{k}^{\alpha}(t)>0\right\}$, then (3.16) with $\bar{E}=\bigcup_{\beta=1}^{m_{\alpha}} \bar{Z}_{k, \beta}^{\alpha, t}$ implies $f_{k}^{\alpha}(t) \leq(2 \pi)^{-1}\left(\frac{d}{d t} f_{k}^{\alpha}(t)\right)^{2}$ because

$$
\begin{aligned}
H^{2}\left(\bigcup_{\beta=1}^{m_{\alpha}} Z_{k, \beta}^{\alpha, t}\right) & \leq H^{2}\left(Y_{k}^{n_{\alpha}, t}\right) \leq \frac{1}{2 \pi}\left(H^{1}\left(\partial Y_{k}^{n_{\alpha}, t}\right)\right)^{2} \leq \frac{1}{2 \pi}\left(H^{1}\left(C_{k}^{n_{\alpha}, t}\right)\right)^{2} \\
& \leq \frac{1}{2 \pi}\left(H^{1}\left(R_{k}^{\alpha} \cap \partial B^{3}(0, t)\right)\right)^{2} \leq \frac{1}{2 \pi}\left(\frac{d}{d t} f_{k}^{\alpha}(t)\right)^{2} .
\end{aligned}
$$

Proceeding as in the proof of Lemma 3 (Filigree lemma) we deduce that

$$
\begin{aligned}
& H^{2}\left(R_{k}^{\alpha} \cap B^{3}(0, t)\right) \leq \varepsilon_{k}^{\alpha}, \\
& \text { whenever } t \leq \rho_{k}-\sqrt{2 / \pi} \sqrt{H^{2}\left(R_{k}^{\alpha} \cap B^{3}\left(0, \rho_{k}\right)\right)} .
\end{aligned}
$$

Now by using (3.8) and (3.16) we have

$$
H^{2}\left(R_{k}^{\alpha} \cap B^{3}\left(0, \rho_{k}\right)\right) \leq H^{2}\left(Y_{k}^{n_{\alpha}}\right)+\varepsilon_{k}^{\alpha} \leq H^{2}\left(\partial B^{3}\left(0, \rho_{k}\right) \cap K_{1, \sigma_{k}}\right)+\varepsilon_{k}^{\alpha} .
$$

Since $H^{2}\left(\partial B^{3}\left(0, \rho_{k}\right) \cap K_{1, \sigma_{k}}\right) \leq 2 \sigma_{k} \Theta^{2}(\mu, p) 2 \pi \rho_{k}$, we deduce

$$
H^{2}\left(R_{k}^{\alpha} \cap B^{3}\left(0, \rho_{k}\right)\right) \leq 4 \pi \rho_{k} \sigma_{k} \Theta^{2}(\mu, p)+\varepsilon_{k}^{\alpha} \leq 5 \pi \sigma_{0} \Theta^{2}(\mu, p)
$$

for sufficiently large $k$ (remember $\frac{3}{4} \sigma_{0}<\sigma_{k}<\sigma_{0}$ and $\rho_{k} \leq 1-\sigma_{k}$ ). Thus (3.22) implies $H^{2}\left(R_{k}^{\alpha} \cap B^{3}(0,3 / 4)\right) \leq \varepsilon_{k}^{\alpha}$ provided $\sigma_{0}$ is sufficiently small. Therefore from (3.14) and (3.15) we have

$$
H^{2}\left(\bigcup_{j=1}^{A_{k}} Q_{k}^{j} \cap B^{3}(0,3 / 4)\right)=\sum_{\alpha=1}^{\bar{L}\left(\rho_{k}\right)} H^{2}\left(R_{k}^{\alpha} \cap B^{3}(0,3 / 4)\right) \leq r^{2} \varepsilon_{k} .
$$

Hence $\left|\bigcup_{j=1}^{A_{k}} Q_{k}^{j} \cap B^{3}(0,3 / 4)\right|$ vanishes as $k \rightarrow \infty$, provided $\sigma_{0}$ is sufficiently small, as claimed above.

Perturbing $\mu_{r}\left(\delta F_{k}^{2}\right)$ slightly, if necessary, we can assume that $\mu_{r}\left(\delta F_{k}^{2}\right)$ is transversal to $\partial B^{3}(0,3 / 4)$. Choose a point $q \in J_{3 / 4,1 / 4}\left(=U^{3}(0,3 / 4) \sim\right.$ $K(3 / 4,1 / 4))$ and let $\pi_{q}$ be the radial projection map from $q$ onto $\partial B^{3}(0,3 / 4)$. Then clearly we have for some $c_{q}<\infty$

$$
H^{2}\left(\pi_{q}\left(\bigcup_{j=1}^{A_{k}} Q_{k}^{j} \cap B^{3}(0,3 / 4)\right)\right) \leq c_{q} H^{2}\left(\bigcup_{j=1}^{A_{k}} Q_{k}^{j} \cap B^{3}(0,3 / 4)\right) .
$$

Since

$$
H^{2}\left(\bigcup_{\alpha=1}^{\bar{L}(3 / 4)} \bigcup_{\beta=1}^{m_{\alpha}} Z_{k, \beta}^{\alpha, 3 / 4}\right) \leq H^{2}\left(\bigcup_{\alpha=1}^{\bar{L}(3 / 4)} Y_{k}^{n_{\alpha}, 3 / 4}\right) \text { and }
$$




$$
\bigcup_{\alpha=1}^{\bar{L}(3 / 4)} Y_{k}^{n_{\alpha}, 3 / 4} \subset \pi_{q}\left(\bigcup_{j=1}^{A_{k}} Q_{k}^{j} \cap B^{3}(0,3 / 4)\right)
$$

we have, by the above inequality,

$$
\lim _{k \rightarrow \infty} H^{2}\left(\bigcup_{\alpha=1}^{\bar{L}(3 / 4)} \bigcup_{\beta=1}^{m_{\alpha}} Z_{k, \beta}^{\alpha, 3 / 4}\right)=0
$$

It follows from (3.9), (3.10), and (3.11) that

$$
\lim _{k \rightarrow \infty}\left|\delta F_{k, L(3 / 4)}^{2,3 / 4}\right|=\lim _{k \rightarrow \infty}\left|\delta F_{k}^{2}\right| \text {. }
$$

Define $F_{k}^{3}=F_{k, L(3 / 4)}^{2,3 / 4}$, which completes the proof of Lemma 7 .

Finally we cut off the filigrees arising from components $Q_{k}^{j}$,s of Case II in the following lemma.

Lemma 8. $\left\{F_{k}^{3}\right\}$ can be replaced by another minimizing sequence $\left\{F_{k}^{4}\right\}$ with the property that $\lim _{k \rightarrow \alpha}\left|\delta F_{k}^{4}\right|=\Delta$, and $\mu_{r}\left(F_{k}^{4}\right) \cap \tilde{B}^{3}(0,3 / 4)$ lies in a bounded set in $\mu_{r}(\tilde{M})$. (That is, every filigree of $\partial\left(\mu_{r}\left(F_{k}^{3}\right)\right)$ in $\mu_{r}(\tilde{M})$ passing through $\tilde{K}_{3 / 4, \sigma_{k}}$ can be cut off.)

Proof of Lemma 8. In this lemma we assume without loss of generality that no component of $\bigcup_{j=A_{k}+1}^{a_{k}} Q_{k}^{j} \cap \partial B^{3}(0, t), 0<t \leq \rho_{k}$, is null-homotopic in $\partial B^{3}(0, t) \cap K_{1, \sigma_{k}}$ since such bad components can be eliminated by cutting (Lemma 2) and slight perturbation, thereby decreasing the area of $\mu_{r}\left(\delta F_{k}^{3}\right)$.

By Corollary 2, the slice of $\mu$ in $\partial B^{3}(0,1)$, as defined in [3, I.3(3)] and denoted by $\langle\mu$, dist, 1$\rangle$, is stationary in $\partial B^{3}(0,1)$ and hence by the structure theorem [2] the number of components of $\partial B^{3}(0,1) \sim \operatorname{spt}\|\mu\|$ is finite, say $c_{p}<\infty$. Therefore $\partial B^{3}(0, t) \sim K_{1, \sigma_{k}}$ has at most $c_{p}$ components for all $t \leq \rho_{k}$. Let $L_{1}, \cdots, L_{d_{k}}, d_{k} \leq c_{p}$, be all components of $\partial B^{3}\left(0, \rho_{k}\right) \sim K_{1, \sigma_{k}}$, and $L_{k, 1}^{j}, \cdots, L_{k, b(j, k)}^{j}$, all components of $\partial B^{3}\left(0, \rho_{k}\right) \sim Q_{k}^{j}$. Then each $L_{m}$, $1 \leq m \leq d_{k}$, is a subset of $L_{k, l}^{j}$ for some $1 \leq l \leq b(j, k)$, and each $\partial L_{k, l}^{j}$ is an individual Jordan curve of the compound Jordan curve $Q_{k}^{j} \cap \partial B^{3}\left(0, \rho_{k}\right)$. Renumbering $L_{k, 1}^{j}, \cdots, L_{k, b(j, k)}^{j}$, if necessary, we can assume that for $\bar{b}(j, k)+$ $1 \leq l \leq b(j, k), L_{k, l}^{j} \subset \partial B^{3}\left(0, \rho_{k}\right) \cap K_{1, \sigma_{k}}$ and for $1 \leq l \leq \vec{b}(j, k), L_{k, l}^{j} \cap$ $\left(\partial B^{3}\left(0, \rho_{k}\right) \sim K_{1, \sigma_{k}}\right) \neq \varnothing$. Hence $\bar{b}(j, k) \leq d_{k} \leq c_{p}$. Let $O_{k, 1}^{j}, \cdots, O_{k, \hat{b}(j, k)}^{j}$ be the set of all components of $B^{3}\left(0, \rho_{k}\right) \sim Q_{k}^{j}, A_{k}+1 \leq j \leq a_{k}$, which are numbered in such a way that $\bar{b}(j, k) \leq \hat{b}(j, k) \leq b(j, k)$ and $\partial B^{3}\left(0, \rho_{k}\right) \cap O_{k, l}^{j} \supset$ $L_{k, l}^{j}$ for $1 \leq l \leq \bar{b}(j, k)$. We then note that, for each $\bar{b}(j, k)+1 \leq l \leq \hat{b}(j, k)$, $Q_{k}^{j} \cap \bar{O}_{k, l}^{j}$ is the image under the projection map $p$ of a filigree of $\partial\left(\mu_{r}\left(\delta F_{k}^{3}\right)\right)$ in $\mu_{r}(\tilde{M})$ passing through $\tilde{K}_{\rho_{k}, \sigma_{k}}$. We are to cut off these filigrees by attaching 
each $O_{k, l}^{j}, \bar{b}(j, k)+1 \leq l \leq \hat{b}(j, k)$, to an appropriate $O_{k, l}^{j}, 1 \leq l \leq \bar{b}(j, k)$. To do so, define $O_{k}^{j}=\bigcup_{l=\bar{b}(j, k)+1}^{\hat{b}(j, k)} \bar{O}_{k, l}^{j}$.

In our cutting and pasting arguments so far, cutting has taken place inside a set $X \subset \subset Y$ whose boundary $\partial X$ is the outermost Jordan curve of $\mu_{r}(\delta F) \cap Y$ (Lemma 2). This time, however, applying the methods of Remark 1, we cut $\mu_{r}\left(p\left(F_{k}^{3}\right)\right)$ along $\partial B^{3}(0, t) \cap O_{k}^{j}, 0<t \leq \rho_{k}$. Let $I^{t}$ be the family of all components of $\left(\partial B^{3}(0, t) \sim Q_{k}^{j}\right) \cap O_{k}^{j}$. First, suppose $X \in I^{t}$ and note that $\mu_{r}\left(p\left(F_{k}^{3}\right)\right) \sim X$ is the union of two disjoint open sets $V_{1}$ and $V_{2}$ which are homeomorphic to $B^{\circ}$. Hence we paste $V_{1}$ to $V_{2}$ along a subset of $\partial V_{1} \cap \partial V_{2} \sim X$ which is a common face of $V_{1}$ and $V_{2}$ other than $X$, to get a new fundamental domain homeomorphic to $B^{\circ}$ (Lemma 5 assures that this pasting does not change the topology of fundamental domain).

Secondly, more generally, $\mu_{r}\left(p\left(F_{k}^{3}\right)\right) \sim \bigcup_{X_{i} \in I_{m}^{t}} X_{i}$ is a disjoint union of open balls for any subfamily $I_{m}^{t}$ of $I^{t}$, and hence it can be pasted appropriately to give a new fundamental domain $F_{k, j, m}^{3, t} \in \Phi$. Here we can arrange this cutting and pasting process in such a way that the resulting fundamental domain $F_{k, j, m}^{3, t} \in \Phi$ satisfies the following properties:

$$
\begin{gathered}
\mu_{r}\left(\delta F_{k, j, m}^{3, t} \sim \mu_{r}\left(\delta F_{k}^{3}\right) \subset \bigcup_{X_{i} \in I_{m}^{t}} X_{i},\right. \\
\mu_{r}\left(\delta F_{k}^{3}\right) \sim \mu_{r}\left(\delta F_{k, j, m}^{3, t}\right) \supset \bar{O}_{k, m}^{j} \cap \partial O_{k}^{j} \cap U^{3}(0, t), 1 \leq m \leq \bar{b}(j, k) .
\end{gathered}
$$

Furthermore it is not difficult to arrange the above cutting and pasting process so that

$$
\bigcup_{m=1}^{\bar{b}(j, k)}\left[\mu_{r}\left(\delta F_{k}^{3}\right) \sim \mu_{r}\left(\delta F_{k, j, m}^{3, t}\right)\right] \supset Q_{k}^{j} \cap O_{k}^{j} \cap U^{3}(0, t) .
$$

Now Lemma 7 implies that $H^{2}\left(\delta F_{k}^{3}\right) \leq H^{2}(\delta G)+\varepsilon_{k}$ for any $G \in \Phi$, where $\varepsilon_{k} \rightarrow 0$ as $k \rightarrow \infty$, so that $H^{2}\left(\mu_{r}\left(\delta F_{k}^{3}\right)\right) \leq H^{2}\left(\mu_{r}\left(\delta F_{k, j, m}^{3, t}\right)\right)+r^{2} \varepsilon_{k}$. Thus

$$
H^{2}\left(\mu_{r}\left(\delta F_{k}^{3}\right) \sim \mu_{r}\left(\delta F_{k, j, m}^{3, t}\right)\right) \leq H^{2}\left(\mu_{r}\left(\delta F_{k, j, m}^{3, t}\right) \sim \mu_{r}\left(\delta F_{k}^{3}\right)\right)+r^{2} \varepsilon_{k} .
$$

Hence (3.23) and (3.25) give

$$
H^{2}\left(Q_{k}^{j} \cap O_{k}^{j} \cap U^{3}(0, t)\right) \leq \bar{b}(j, k)\left(H^{2}\left(\bigcup_{X_{i} \in I_{m}^{t}} X_{i}\right)+r^{2} \varepsilon_{k}\right) .
$$

Since $\bar{b}(j, k) \leq c_{p}$ we can replace $\bar{b}(j, k)$ by $c_{p}$ in (3.26).

Define

$$
f_{k}^{j}(t)=H^{2}\left(Q_{k}^{j} \cap O_{k}^{j} \cap U^{3}(0, t)\right)-c_{p} r^{2} \varepsilon_{k}, t \in\left[t_{k}^{j}, \rho_{k}\right], t_{k}^{j}=\inf \left\{t: f_{k}^{j}(t)>0\right\} .
$$


Then by (3.26) we have $f_{k}^{j}(t) \leq c_{p} H^{2}\left(\bigcup_{X_{i} \in I_{m}^{t}} X_{i}\right)$. From the isoperimetric inequality on $\partial B^{3}(0, t)$ and the co-area formula it follows that

$$
f_{k}^{j}(t) \leq \frac{c_{p}}{2 \pi}\left(\frac{d}{d t} f_{k}^{j}(t)\right)^{2} \quad \text { a.e. } t \in\left[t_{k}^{j}, \rho_{k}\right] .
$$

By applying the same method as in Lemma 3 (Filigree lemma) we obtain

$$
H^{2}\left(Q_{k}^{j} \cap O_{k}^{j} \cap U^{3}(0, t)\right) \leq c_{p} r^{2} \varepsilon_{k}
$$

whenever

$$
t \leq \rho_{k}-\sqrt{2 c_{p} / \pi} \sqrt{H^{2}\left(Q_{k}^{j} \cap O_{k}^{j} \cap U^{3}\left(0, \rho_{k}\right)\right.} .
$$

Now using (3.26) we get

$$
H^{2}\left(Q_{k}^{j} \cap O_{k}^{j} \cap U^{3}\left(0, \rho_{k}\right)\right) \leq c_{p} H^{2}\left(K_{1, \sigma_{k}} \cap \partial B^{3}\left(0, \rho_{k}\right)\right)+c_{p} r^{2} \varepsilon_{k} .
$$

Hence $H^{2}\left(Q_{k}^{j} \cap O_{k}^{j} \cap U^{3}\left(0, \rho_{k}\right)\right) \leq 4 \pi c_{p} \rho_{k} \sigma_{k} \Theta^{2}(\mu, p)+c_{p} r^{2} \varepsilon_{k}$. Therefore for sufficiently large $k$, we have

$$
H^{2}\left(Q_{k}^{j} \cap O_{k}^{j} \cap U^{3}\left(0, \rho_{k}\right)\right) \leq 5 \pi c_{p} \sigma_{0} \Theta^{2}(\mu, p) .
$$

Thus we obtain $H^{2}\left(Q_{k}^{j} \cap O_{k}^{j} \cap U^{3}(0,3 / 4)\right) \leq c_{p} r^{2} \varepsilon_{k}$, provided $\sigma_{0}$ is sufficiently small. Hence $\left|Q_{k}^{j} \cap O_{k}^{j} \cap U^{3}(0,3 / 4)\right|$ vanishes as $k \rightarrow \infty$. Then, by using the projection map $\pi_{q}$ as in the proof of Lemma 7 , we see that

$$
H^{2}\left(O_{k}^{j} \cap \partial B^{3}(0,3 / 4)\right) \rightarrow 0 \quad \text { as } k \rightarrow \infty .
$$

By the assumption at the beginning of this proof each component of

$$
\left(\bigcup_{j} O_{k}^{j} \cap \partial B^{3}(0,3 / 4)\right) \sim \mu_{r}\left(\delta F_{k}^{3}\right)
$$

is homeomorphic to $D$. Hence $\mu_{r}\left(p\left(F_{k}^{3}\right)\right) \sim\left(\bigcup_{j} O_{k}^{j} \cap \partial B^{3}(0,3 / 4)\right) \approx \bigcup_{i} V_{i}$, $V_{i} \approx B^{\circ}$. Now, pasting $\bigcup_{j} O_{k}^{j} \cap U^{3}(0,3 / 4)$ to $U^{3}(0,3 / 4) \sim \bigcup_{j} O_{k}^{j}$ in an appropriate way and performing, if necessary, more pastings inside $\mu_{r}(M) \sim$ $U^{3}(0,3 / 4)$, we can get $F_{k}^{4} \in \Phi$ such that

$$
\begin{gathered}
\mu_{r}\left(\delta F_{k}^{4}\right) \sim \mu_{r}\left(\delta F_{k}^{3}\right) \subset \bigcup_{j} O_{k}^{j} \cap \partial B^{3}(0,3 / 4), \\
\left(\mu_{r}\left(\delta F_{k}^{4}\right) \cap B^{3}\left(0, \frac{3}{4}\right)\right) \cup\left(\bigcup_{j} O_{k}^{j} \cap \partial B^{3}\left(0, \frac{3}{4}\right)\right)
\end{gathered}
$$

encloses no domain in $U^{3}\left(0, \frac{3}{4}\right)$. Note however that $\left(\mu_{r}\left(\delta F_{k}^{3}\right) \cap B^{3}(0,3 / 4)\right) \cup$ $\left(\bigcup_{j} O_{k}^{j} \cap \partial B^{3}(0,3 / 4)\right)$ encloses domains which are subsets of $\bigcup_{j} O_{k}^{j} \cap B^{3}(0,3 / 4)$. 
By (3.27) and (3.28) we have $\lim _{k \rightarrow \infty}\left|\delta F_{k}^{4}\right|=\lim _{k \rightarrow \infty}\left|\delta F_{k}^{3}\right|$. Thus we see that no spike, or filigree, of $\partial\left(\mu_{r}\left(F_{k}^{4}\right)\right)$ in $\mu_{r}(\tilde{M})$ can come into $\tilde{U}^{3}(0,3 / 4)$. Hence $B^{3}(0,3 / 4) \sim \mu_{r}\left(\delta F_{k}^{4}\right)$ has only finite components, and therefore $\mu_{r}\left(F_{k}^{4}\right) \cap$ $\tilde{B}^{3}(0,3 / 4)$ lies in a bounded set in $\mu_{r}(\tilde{M})$. This completes the proof of Lemma 8.

Finally we are in a position to finish the proof of Theorem 2. In Lemma 8 $r$ depends on $p$, say, $r=r_{p}$. By compactness of $M$ there exists a set $I$ of a finite number of points in $M$ such that $\left\{U^{3}\left(q, 3 /\left(4 r_{q}\right)\right\}_{q \in I}\right.$ covers $M$. Hence, applying Lemma 8 repeatedly at all points of $I$, we deduce that $\left\{F_{k}^{4}\right\}$ must be uniformly bounded in $\tilde{M}$ provided $\bigcap_{k} F_{k}^{4} \neq \varnothing$.

Now remember that we assumed at the beginning of the proof of Theorem 2 that $M$ is locally isometrically in $\mathbf{R}^{3}$. However we can easily see that if $M$ is not locally in $\mathbf{R}^{3}$, all the methods we have used so far are directly applicable to the images under the exponential map (i.e., exponential image of the tangent cone $\mu$, exponential image of $K_{\rho, \sigma}$, etc). Hence the proof is complete.

The following corollary says that $\Delta\left(=\lim _{k \rightarrow \infty}\left|\delta F_{k}\right|\right)$ is an area minimizing integral varifold in $M$ and is regular in a neighborhood of any point of spt $\|\Delta\|$ where there is a varifold tangent with support contained in a plane. (By rectifiability there is such a tangent plane at almost all points of spt $\|\Delta\|$.)

Corollary 3 (a.e. smoothness). If $\Delta$ is the varifold limit of a minimizing sequence in $\Phi_{0}$ ( or $\left.\Phi\right)$, and has a varifold tangent $\mu$ at $p \in \operatorname{spt}\|\Delta\|$ with spt $\|\mu\| \subset H$, where $H$ is a plane, then there is an $r>0$ such that $\|\Delta\|_{\llcorner} B^{3}(p, r)=\|n|S|\|$, where $n$ is a positive integer and $S$ is a smooth (analytic if the metric of $M$ is analytic) oriented connected minimal surface containing $p$.

Proof. Since spt $\|\mu\|$ is a plane we deduce from (3.29) that each component of $\mu_{r}\left(\delta F_{k}^{4}\right) \cap B^{3}(0,3 / 4)$ is homeomorphic to a disk, and hence each component of $\mu_{r}\left(\delta F_{k}^{4}\right) \cap \partial B^{3}(0,3 / 4)$ is a circle which is not null-homotopic in $\partial B^{3}(0,3 / 4) \cap K_{1, \sigma_{k}}$. Then by using the arguments in [4, Theorem 2], we conclude the required result.

\section{Fundamental domains with least boundary area}

Since now we have a uniformly bounded minimizing sequence of fundamental domains, we can show the existence and regularity of fundamental domains of $M$ which minimize boundary area among all fundamental domains in $\Phi_{0}$.

Given a continuous map $g$ on $M$ we define $\tilde{g}$ to be a map on $\tilde{M}$ satisfying $p \tilde{g}=g p$. Of course $\tilde{g}$ is not unique, but its uniqueness is not necessary in our 
setting. For a function $h$ on $\tilde{M}$ we define the function $h^{M}$ on $M$ by

$$
h^{M}(x)=\sum_{y \in p^{-1}(x)} h(y), \quad x \in M
$$

Thus $h^{M}$ is well defined only for a restricted family of functions $h$ on $\tilde{M}$. If $F$ is a fundamental domain of $M$, then $\left(\chi_{F}\right)^{M}=1$ almost everywhere in $M$, where $\chi_{F}$ is the characteristic function of $F$ on $\tilde{M}$. Also if $H^{2}(\partial F)<\infty$, then we can easily see that $\chi_{F}$ is a $B V$ function on $\tilde{M}$. Since $\partial^{*} F$ represents "actual" boundary of $F$, we may think of $\left|D \chi_{F}\right|(\tilde{M})$ as the boundary area of $F\left(\right.$ recall $\left.\left|D \chi_{F}\right|(\tilde{M})=H^{2}\left(\partial^{*} F\right)\right)$.

Let $I=\inf \left\{H^{2}\left(\partial^{*} F\right): F \in \Phi_{0}\right\}$ and let $\left\{F_{k}\right\}$ be a sequence of fundamental domains in $\Phi_{0}$ such that $H^{2}\left(\partial^{*} F_{k}\right) \rightarrow I$. Then by compactness of $B V$ functions $[11,6.3]$ there are a subsequence $\left\{\chi_{F_{k}^{\prime}}\right\} \subset\left\{\chi_{F_{k}}\right\}$ and a $B V$ function $u$ on $\tilde{M}$ such that

$$
\chi_{F_{k}^{\prime}} \rightarrow u \quad \text { in } L_{\text {loc }}^{1}(\tilde{M}), \quad|D u|(\tilde{M}) \leq \lim \inf \left|D \chi_{F_{k}^{\prime}}\right|(\tilde{M}) \quad(=I) .
$$

$u$ is obviously a characteristic function $\chi_{F}$ of some set $F$ on $\tilde{M}$. By Theorem 2 the sequence $\left\{F_{k}^{\prime}\right\}$ can be assumed to be uniformly bounded in $\tilde{M}$. Hence

$$
1=\left(\chi_{F_{k}^{\prime}}\right)^{M} \rightarrow\left(\chi_{F}\right)^{M} \text { in } L_{\text {loc }}^{1}(M) .
$$

It follows that $F$ itself is a fundamental domain of $M$ and $H^{2}\left(\partial^{*} F\right)=I$.

Now the following questions about $F$ arise:

(a) Is $p\left(\partial^{*} F\right)$ locally area minimizing under a Lipschitz map on $M$ ?

(b) Is $F$ connected?

If both questions are answered affirmatively, then $F$ is the desired fundamental domain with least boundary area.

Question (a). Let $k$ be a $C^{1}$ map on $\tilde{M}$ (or $M$ ), and $h$ a function on $\tilde{M}$ (or $M$ ). Define the function $h^{k}$ on $\tilde{M}$ (or $M$ ), by

$$
h^{k}(x)=\sum_{y \in k^{-1}(x)} \omega(y) h(y)
$$

where

$$
\omega(y)= \begin{cases}1 & \text { if } k \text { is orientation-preserving at } y, \\ -1 & \text { if } k \text { is orientation-reversing at } y, \\ 0 & \text { if } J k(y)=0, \text { where } J k \text { is the Jacobian of } k .\end{cases}
$$

Note that even if $k$ is Lipschitz, $h^{k}$ is defined almost everywhere. Let $g$ be a Lipschitz map on $M$ such that $\{x: g(x) \neq x\} \cup g\{x: g(x) \neq x\}$ is contained in a small ball in $M$. Then by the above definition it is not difficult to check 
that $\left(\left(\chi_{F}\right)^{\tilde{g}}\right)^{M}=\left(\left(\chi_{F}\right)^{M}\right)^{g}=1^{g}=1$ a.e. on $M$, and therefore also to show that there exists a fundamental domain $F_{g}$ of $M$ such that

$$
\operatorname{spt}\left|D \chi_{F_{g}}\right| \subset \operatorname{spt}\left|D\left(\chi_{F}\right)^{\tilde{g}}\right| \text {. }
$$

Since $\operatorname{spt}\left|D\left(\chi_{F}\right)^{\tilde{g}}\right|=\tilde{g}\left(\operatorname{spt}\left|D \chi_{F}\right|\right)=\tilde{g}\left(\partial^{*} F\right)$, we have

$$
H^{2}\left(p\left(\partial^{*} F_{g}\right)\right) \leq H^{2}\left(p\left(\tilde{g}\left(\partial^{*} F\right)\right)\right) \quad\left(=H^{2}\left(g\left(p\left(\partial^{*} F\right)\right)\right)\right),
$$

or $H^{2}\left(p\left(\partial^{*} F\right)\right) \leq H^{2}\left(g\left(p\left(\partial^{*} F\right)\right)\right)$. Therefore $p\left(\partial^{*} F\right)$ is locally area minimizing.

Note. J. Taylor's arguments in [13] might be true even if one assumes that all the Lipschitz maps in [13] are nowhere orientation-reversing. In this case the interior of $\tilde{g}(F)$ is clearly a fundamental domain of $M$.

Question (b). Suppose $F$ is not connected. Then there are two components $U, V$ of $F$ and subsets $X, Y$ of $\partial U, \partial V$ respectively such that $p(U) \cap p(V)=p(X)=p(Y)$ and $H^{2}(p(X))>0$. Hence there must exist a translation $\tau$ on $\tilde{M}$ for which $\tau(X)=Y$. It follows that $(F \sim U) \cup \tau(U)$ is a fundamental domain with less boundary area than $F$. This contradiction proves the connectedness of $F$.

Let $\nu$ be a diffeomorphism from $B^{3}(p, r) \subset M, p \in \delta F$, to $B^{3}(0, r) \subset$ $\mathbf{R}^{3}$, and let $\mathbf{F}$ be the measure over $\mathbf{R}^{3}$ corresponding to $H^{2}$ over $M$ under $\nu$, i.e., $\mathbf{F}(\nu(S))=H^{2}(S)$ for any $H^{2}$ measurable subset $S$ of $M$. Then $\nu(\delta F)\left(=\nu\left(p\left(\partial^{*} F\right)\right)\right)$ is locally $\mathbf{F}$-minimizing under Lipschitz deformation in the sense that for any Lipschitz map $\phi$ on $M$ with $\{x: \phi(x) \neq x\} \cup$ $\phi\{x: \phi(x) \neq x\} \subset B^{3}(p, r)$ we have

$$
\mathbf{F}(\nu(\delta F)) \leq \mathbf{F}(\nu(\phi(\delta F))) .
$$

On the other hand one can find a function $\xi(r)=C r^{\alpha}$ with $0 \leq C<\infty$ and $0<\alpha<1 / 3$ such that

$$
H^{2}(\nu(\delta F \cap W)) \leq(1+\xi(r)) H^{2}(\nu(\phi(\delta F \cap W))),
$$

where $W=\{x: \phi(x) \neq x\}$ and $r=\operatorname{diam}(W \cup \phi(W))$. Hence $\nu(\delta F)$ is $(\mathbf{M}, \xi, \delta)$ minimal as defined in $[13$, I. (8)].

Thus by [13, II.4, II.6, IV.5, IV.8] we get the following theorem.

Theorem 3. There exists a fundamental domain $F \in \Phi_{0}$ with least boundary area among all elements of $\Phi_{0}$. Moreover,

(i) $\delta F=R(\delta F) \cup \sigma_{Y}(\delta F) \cup \sigma_{T}(\delta F)$;

(ii) $\sigma_{T}(\delta F)$ consists of isolated points;

(iii) $\sigma_{Y}(\delta F)$ is a one-dimensional $C^{1, \alpha}$ submanifold;

(iv) $R(\delta F)$ is a smooth minimal surface; 
(v) for every $p \in \sigma_{Y}(\delta F)$ (respectively $\sigma_{T}(\delta F)$ ) there is a neighborhood $N$ of $p$ and $a C^{1, \alpha / 2}$ diffeomorphism $f: B \rightarrow N$ such that $\delta F \cap N=f(Y)$ (respectively $f(T)$ ).

Remark 2. Suppose $\hat{M}$ is a covering space of $M$ which is not necessarily the universal covering space $\tilde{M}$ of $M$. We define a "fundamental domain" $F$ of $M$ in $\hat{M}$ as we did in $\tilde{M}$. Then we can again conclude that there exists a fundamental domain $F$ in $\hat{M}$ with least boundary area and satisfying the regularity results (i)-(v) of Theorem 3 . Obviously the proof of Theorem 3 remains valid for fundamental domains in $\hat{M}$.

As for the two-dimensional compact Riemannian manifold $M^{2}$, we get the following proposition concerning the existence, regularity, and topology of fundamental domains with least boundary length.

Proposition. There exists a fundamental domain $F$ of $M^{2}$ which minimizes boundary length among all fundamental domains of arbitrary topological type with the properties that

(i) $\bar{F}$ is homeomorphic to a closed disk,

(ii) $F$ is a polygon such that the edges of $F$ are geodesic segments in $\tilde{M}$, interior angles of vertices of $F$ are $120^{\circ}$, and if $\chi\left(M^{2}\right) \leq 0$ then the number of vertices of $F$ ( $=$ the number of edges of $F$ ) is equal to $6-6 \chi\left(M^{2}\right)$.

Proof. Note that a minimizing sequence of connected fundamental domains of $M^{2}$ must be uniformly bounded if they have nonempty intersection. Therefore we can proceed as above, using characteristic functions of fundamental domains, to conclude that there exists a connected fundamental domain $F$ with least boundary area. Suppose $\bar{F}$ is multiply connected, and $J_{1} \approx D$ is a component of $\tilde{M}^{2} \sim \bar{F}$ with $H^{2}\left(J_{1}\right)<\infty$. Then there exists a translation $\tau_{1}$ on $\tilde{M}^{2}$ such that $\tau_{1}(F) \subset J_{1}$. Since $\tau_{1}(\bar{F})$ is multiply connected, we have a component $J_{2} \approx D$ of $J_{1} \sim \tau_{1}(\bar{F})$ and a translation $\tau_{2}$ such that $\tau_{2}(F) \subset J_{2}$. Continuing this process we can get translations $\tau_{1}, \tau_{2}, \tau_{3}, \cdots$ such that the fundamental domains $\tau_{1}(F), \tau_{2}(F), \tau_{3}(F), \cdots$ are subsets of $J_{1}$ and pairwise disjoint. This is not possible since $H^{2}\left(J_{1}\right)<\infty$. Therefore $\bar{F} \approx \bar{D}$.

The first part of (ii) follows from the fact that $\delta F$ is locally area minimizing under a Lipschitz map. Now we note that a straight line and $Y^{1}$ are the only area minimizing (under the Lipschitz map) 1-varifolds up to rotation (see [13, II.3.]). Hence we deduce the second part of (ii). For the third part of (ii) we recall the Gauss-Bonnet formula,

$$
\int_{F} K d A=-\int_{\partial F} \kappa_{g} d s+\sum_{i=1}^{n}\left(\alpha_{i}-\pi\right)+2 \pi,
$$

where $K$ is the Gaussian curvature of $\hat{M}$ (or $M$ ), $\kappa_{g}$ is the signed geodesic curvature of $\partial F$, and $\alpha_{1}, \cdots, \alpha_{n}$ are the interior angles of vertices of $F$. 
Since $\int_{F} K d A=\int_{M^{2}} K d A=2 \pi \chi\left(M^{2}\right)$ and $\kappa_{g}=0$, we obtain $2 \pi \chi\left(M^{2}\right)=$ $-n \pi / 3+2 \pi$, which gives the last part of (ii).

\section{Regularity of singular set}

In this section we show the existence and regularity of a fundamental domain in $\Phi$ with least boundary area. Without loss of generality we assume, in Lemma 9 and Corollary 4 , that $M$ is locally isometrically in $\mathbf{R}^{3}$ and that for any $p \in \operatorname{spt}\|\Delta\|$ we choose $p$ to be the origin in $\mathbf{R}^{3}$.

So far we have not ruled out the possibilities that $\mu_{r}\left(\delta F_{k}^{4}\right) \cap B^{3}(0,3 / 4)$ might have more than one component, and that the tangent cone $\mu$ of $\Delta$ at $p \in$ spt $\|\Delta\|$ might be other than $|D|,|Y|$, or $|T|$. The following lemma rules out these possibilities.

Lemma 9. For any $p \in \operatorname{spt}\|\Delta\|$ the tangent cone $\mu$ of $\Delta$ at $p$ is $|D|,|Y|$, or $|T|$.

Proof. Suppose $\mu_{r}\left(\delta F_{k}^{4}\right) \cap B^{3}(0,3 / 4)$ has more than one component for sufficiently large $k$. Then we can find a component $Q_{k}$ of $\mu_{r}\left(\delta F_{k}^{4}\right) \cap B^{3}(0,3 / 4)$ for each $k$ and a cone $\tau$ such that $\lim _{r \rightarrow \infty, k \rightarrow \infty}\left|Q_{k}\right|=\tau$, spt $\|\tau\| \subset$ spt $\|\mu\|$, and $\tau \neq \mu$. If spt $\|\tau\|$ were not a plane, then we could construct a diffeomorphism of $B$ fixing $\partial B$ which would decrease not only the mass of $\tau$ but also the area of $\mu_{r}\left(\delta F_{k}^{4}\right)$ for large $r$ and $k$, an obvious contradiction. Thus we conclude that if $\mu_{r}\left(\delta F_{k}^{4}\right) \cap B^{3}(0,3 / 4)$ has more than one component for large $k$, then $\mu=m|D|, m>1$.

Next assume that $\mu_{r}\left(\delta F_{k}^{4}\right) \cap B^{3}(0,3 / 4)$ is connected for large $k$. Then we know from the construction of $\mu_{r}\left(\delta F_{k}^{4}\right)$ (Lemma 8) that $\mu=\mid$ spt $\|\mu\| \mid$. Assume that spt $\|\mu\| \neq D, Y, T$. First, if spt $\|\mu\|$ is one of those seven cones which are proven to be not area minimizing under Lipschitz deformation in $[13$, II.3], then we note that every Lipschitz map $\psi$ constructed in [13, II.3] for each non-area-minimizing cone $\nu$ satisfies the property that each component of $U^{3}(0,1) \sim \psi(\operatorname{spt}\|\mu\|)$ is homeomorphic to $B^{\circ}$ and

$$
H^{2}\left(\psi(\text { spt }\|\nu\|) \cap B^{3}(0,1)\right)<H^{2}\left(\operatorname{spt}\|\nu\| \cap B^{3}(0,1)\right) .
$$

Hence it follows that for large $r$ and $k$, we can similarly construct the Lipschitz map $\psi$ on $\mu_{r}(M)$ which leaves $\mu_{r}(M) \sim B^{3}(0,1)$ fixed such that

$$
\left\{\begin{array}{l}
\mu_{r}(M) \sim \psi\left(\mu_{r}\left(\delta F_{k}^{4}\right)\right) \approx B^{\circ} \text { and } \\
H^{2}\left(\psi\left(\mu_{r}\left(\delta F_{k}^{4}\right)\right)\right)<\mathbf{M}\left(\mu_{r \#} \Delta\right) \leq H^{2}\left(\mu_{r}\left(\delta F_{k}^{4}\right)\right),
\end{array}\right.
$$

an obvious contradiction. Second, if spt $\|\mu\|$ is different from those cones of $\left[13\right.$, II.3], that is, its intersection with $\partial B^{3}(0,1)$ is a 1-varifold with multiple points other than triple point (i.e., quadruple point, etc.), then, in view of a 
Lipschitz map on $\partial B^{3}(0,1)$ which squashes a quadruple point to become two triple points and decreases the length of spt $\|\mu\| \cap \partial B^{3}(0,1)$, we can construct $\psi$ satisfying (5.1) more easily than in the above case. Therefore spt $\|\mu\|$ must be equal to $D, Y$, or $T$, and hence, we deduce that $\mu=|D|,|Y|$, or $|T|$ in case $\mu_{r}\left(\delta F_{k}^{4}\right) \cap B^{3}(0,3 / 4)$ is connected for $k$ large.

We now note that by Corollary 3 the density of $R(\Delta)$ is constant in a component of $R(\Delta)$, and the boundary of this component contains the points of $\sigma_{Y}(\Delta)$. Hence we deduce that if $\mu=m|D|$ along a component of $R(\Delta)$, then $m$ must be equal to 1 . Therefore we conclude that $\mu_{r}\left(\delta F_{k}^{4}\right) \cap B^{3}(0,3 / 4)$ is connected for large $k$, and $\mu=|D|,|Y|$, or $|T|$.

The following corollary and its proof are almost similar to [13, II.6].

Corollary 4 ( $C^{0}$ regularity of multiple curve). (1) $\sigma_{T}(\Delta)$ consists of isolated points.

(2) $\sigma_{Y}(\Delta)$ is a one-dimensional $C^{0}$ submanifold.

(3) Suppose $p \in \sigma_{Y}(\Delta)$ (resp. $\sigma_{T}^{\prime}(\Delta)$ ). Then for some $r>0, R(\Delta) \cap$ $B^{3}(p, r)$ consists of three (resp. six) components, each of which is a smooth manifold.

Proof. (1) This conclusion follows from monotonicity (Lemma 4) and the weak convergence to tangent cones as varifolds.

(2) It is not difficult to see that Lemma II.5 of [13] holds in our setting with $\mu_{i}(\mathrm{spt}\|\Delta\|)$ in place of $S_{i}$. Hence

$$
H D\left(\sigma_{Y}\left(\mu_{r} \tau_{p}\left(\operatorname{spt}\|\Delta\| \cap B^{3}(p, 3 / 4)\right)\right), \sigma_{Y}(\phi(Y))\right)
$$

(for some $\phi \in \mathrm{SO}(3)$ depending on $p$ ) goes to zero as $r$ goes to $\infty$ for each fixed $p \in \sigma_{Y}(\Delta)$ and is uniformly small as a function of $p$ in small compact subsets of $\sigma_{Y}(\Delta)$ for fixed $r>0$. Therefore the set $\sigma_{Y}(\Delta)$ satisfies Reifenberg's condition in $\S 4$ of [10], and is hence a one-dimensional $C^{0}$ submanifold.

(3) This follows from Corollary 3 and [13, II.6(4)].

An epiperimetric inequality is an inequality which gives us an upper bound to the area of area minimizing surface. This upper bound of area gives us $C^{1, \alpha}$ regularity of area minimizing surface at its singular set. The statement and the proof of epiperimetric inequality basically follow [13]. Before stating the following lemma we should note that the competing surfaces of $\Delta$ are not only the images of $\Delta$ under diffeomorphisms but also all the varifolds $\Gamma$ with $\Gamma=\lim _{k \rightarrow \infty}\left|\delta F_{k}^{\prime}\right|, F_{k}^{\prime} \in \Phi$. We note also that $M$ is no longer assumed locally isometric to $\mathbf{R}^{3}$. However, using the diffeomorphism from $B^{3}(p, r) \subset M$ to $B^{3}(0, r) \subset \mathbf{R}^{3}$, we equip $M \cap B^{3}(p, r)$ with the metric $\nu^{*} g$, where $g$ is the Euclidean metric of $\mathbf{R}^{3}$.

Lemma 10 (Epiperimetric inequality for $\Delta$ ). There exist $\varepsilon>0, \varsigma>0$, and $k>0$, such that if 
(i) $p \in \sigma_{Y}(\Delta)\left(\right.$ respectively $\left.\sigma_{T}(\Delta)\right)$;

(ii) for some $r>0$,

$$
r^{-2} H^{2}\left(\operatorname{spt}\|\Delta\| \cap B^{3}(p, r)\right)-\pi \Theta^{2}(\Delta, p)<\varepsilon,
$$

and

$$
H D\left(\mu_{1 / r} \tau_{p}\left(\operatorname{spt}\|\Delta\| \cap B^{3}(p, r)\right), \theta Y\right)<\varsigma
$$

(respectively, replace $\theta Y$ by $\theta T$ ) for some $\theta \in \mathrm{SO}(3)$, then there exists a 2varifold $\Gamma$ with $\Gamma=\lim _{k \rightarrow \infty}\left|\delta F_{k}^{\prime}\right|$ for a sequence $\left\{F_{k}^{\prime}\right\}$ in $\Phi$ such that

(1) $\operatorname{spt}\|\Gamma\| \sim B^{3}(p, r) \subset \operatorname{spt}\|\Delta\| \sim B^{3}(p, r)$ and

$$
\begin{aligned}
H^{2}\left(\operatorname{spt}\|\Gamma\| \cap B^{3}(p, r)\right) \leq & (1-k / 2)(r / 2) H^{1}\left(\operatorname{spt}\|\Delta\| \cap \partial B^{3}(p, r)\right) \\
& +(k / 2) \pi r^{2} \Theta^{2}(\Delta, p) .
\end{aligned}
$$

Proof. First, we prove the lemma under the assumption that spt $\|\Delta\| \cap$ $\partial B^{3}(p, r)$ consists of a finite number of Lipschitz curves and is homeomorphic to $Y \cap \partial B$ (resp. $T \cap \partial B$ ). Define $J=\operatorname{spt}\|\Delta\| \cap \partial B^{3}(p, r)$. Let $\left\{\phi_{n}\right\}$ be a sequence of shrinking diffeomorphisms in $B^{3}(p, r)$ with $\phi_{n}(x)=x, x \in$ $\partial B^{3}(p, r)$ for all $n$ such that $\lim _{n \rightarrow \infty} \phi_{n \#} \Delta=|p \star J|$. From [12, Chapter 3] and $[13$, III.5] one observes that there exist $\varepsilon>0, \varsigma>0$, and $k>0$ such that if (i) and (ii) are satisfied then one can find a diffeomorphism $\psi$ with $\{x: \psi(x) \neq x\} \cup \psi\{x: \psi(x) \neq x\} \subset B^{3}(p, r)$ such that

$$
\left.H^{2}(\psi(p) J)\right) \leq(1-k)(r / 2) H^{1}(J)+k \pi r^{2} \Theta^{2}(\Delta, p) .
$$

In fact, the Lipschitz maps in [12, Chapter 3] can be replaced by diffeomorphisms since the Lipschitz maps there count multiplicity of area. Since by $[3$, I.2.6] the mapping of the sequence of varifolds $\left\{\phi_{n \#} \Delta\right\}$ by $\psi$ is continuous, we have

$$
\lim _{n \rightarrow \infty} \psi_{\#} \phi_{n \#} \Delta=\psi_{\#} \lim _{n \rightarrow \infty} \phi_{n \#} \Delta=\psi_{\#} \mid p \text { 咴J|. }
$$

Hence

$$
\lim _{n \rightarrow \infty}\left\|\left(\psi \phi_{n}\right)_{\#} \Delta\right\|\left(U^{3}(p, r)\right) \leq(1-k)(r / 2) H^{1}(J)+k \pi r^{2} \Theta^{2}(\Delta, p) .
$$

Therefore for sufficiently large $n$,

$$
\left\|\left(\psi \phi_{n}\right)_{\#} \Delta\right\|\left(U^{3}(p, r)\right) \leq\left(1-\frac{3}{4} k\right)(r / 2) H^{1}(J)+\frac{3}{4} k \pi r^{2} \Theta^{2}(\Delta, p) .
$$

This proves conclusion (2) with $k$ replaced by $\frac{3}{4} k, \Gamma=\left(\psi \phi_{n}\right)_{\#} \Delta, F_{k}^{\prime}=\tilde{\psi} \tilde{\phi}_{n} F_{k}$, and conclusion (1) follows from the choice of $\psi$ and $\phi_{n}$.

Secondly, we prove the lemma under the assumption that spt $\|\Delta\| \cap \partial B^{3}(p, r)$ $(=J)$ consists of a finite number of Lipschitz curves. Let $K$ be the subset of $J$ as defined in [13, III.4] such that $K$ is homeomorphic to $Y \cap \partial B$ (resp. 
$T \cap \partial B$ ). Then we get open components $U^{1}, U^{2}, U^{3}$ (resp. $U^{1}, \cdots, U^{4}$ ) of $U^{3}(p, r) \sim(p * K)$. Now we apply a replacement argument as in Theorem 1 to each $U^{l}$ and $F_{k}$. For this lemma we make one alteration to the proof of Theorem 1. For each $U^{l}$ we define $K_{i}^{l}, C_{i j}^{l}$ as in Theorem 1 . But we let $X_{i j 1}^{l}$ be the component of $\partial U^{l} \sim C_{i j}^{l}$ which contains the set $Z^{l}=\left\{x \in \partial U^{l}: \operatorname{dist}(x, p * K)>\varsigma\right\}$. Then we define $Y_{i j}^{l}=\partial U^{l} \sim \bar{X}_{i j 1}^{l}$ and proceed from here exactly as in Theorem 1 . Hence we get $F_{k}^{0}\left(=F_{k}\right), F_{k}^{1}, F_{k}^{2}$, $F_{k}^{3}\left(\right.$ resp. $\left.F_{k}^{0}, \cdots, F_{k}^{4}\right) \in \Phi$ with

$$
\left.\delta F_{k}^{l} \sim U^{l} \subset \delta F_{k}^{l-1} \sim U^{l}, \quad l=1,2,3 \text { (resp. } 1, \cdots, 4\right) .
$$

Also, if we define $U_{\eta}^{l}=\left\{x \in U^{l}: \operatorname{dist}\left(x, \partial U^{l}\right)<\eta\right\}$, we can arrange replacement procedure in such a way that $\delta F_{k}^{l} \cap U^{l} \subset U_{\eta}^{l}$ for any $\eta>0$. Hence letting $\eta \rightarrow 0$ we obtain

$$
\text { spt }\left\|\lim _{k \rightarrow \infty}\left|\delta F_{k}^{l} \cap U^{l}\right|\right\| \subset \partial U^{l} .
$$

Moreover we may assume that for each component $E$ of $\delta F_{k}^{l} \cap U^{l}, H^{2}(E)$ is arbitrarily close to $H^{2}(Z)$, where $Z$ is the subset of $\partial U^{l}$ with $\partial Z=\partial E$ and $Z \cap Z^{l}=\varnothing$. Then it follows from (5.2) that since $\Delta$ has density 1 almost everywhere the varifold $\lim _{k \rightarrow \infty}\left|\delta F_{k}^{l}\right|$ also has density 1 almost everywhere for each $l$.

Let $\Delta^{\prime}=\lim _{k \rightarrow \infty}\left|\delta F_{k}^{3}\right|$ (resp. $\left.\lim _{k \rightarrow \infty}\left|\delta F_{k}^{4}\right|\right)$. Note that the boundary of spt $\left\|\Delta^{\prime}\right\| \cap U^{3}(p, r)$ is $K$. Hence the arguments of the first case is applicable to spt $\left\|\Delta^{\prime}\right\| \cap U^{3}(p, r)$ and therefore there exist diffeomorphisms $\phi_{n}$ and $\psi$ such that

$$
\left\|\left(\psi \phi_{n}\right)_{\#} \Delta^{\prime}\right\|\left(U^{3}(p, r)\right) \leq\left(1-\frac{3}{4} k\right)(r / 2) H^{1}(K)+\frac{3}{4} k \pi r^{2} \Theta^{2}(\Delta, p) .
$$

On the other hand, [13, III.4(4)] implies

$$
H^{2}\left(\text { spt }\left\|\Delta^{\prime}\right\| \cap \partial B^{3}(p, r)\right) \leq 25\left[H^{1}(J)-H^{1}(K)\right]^{2} .
$$

We note however that for any set $E$ in a thin strip with width $2 \varsigma r$ we can use an isoperimetric inequality $H^{2}(E) \leq \varsigma r H^{1}(\partial E)$ instead of $H^{2}(E) \leq\left[H^{1}(\partial E)\right]^{2}$. Hence (5.4) can be replaced by

$$
H^{2}\left(\mathrm{spt}\left\|\Delta^{\prime}\right\| \cap \partial B^{3}(p, r)\right) \leq 5 \zeta r\left[H^{1}(J)-H^{1}(K)\right] .
$$

Since $\left|\operatorname{spt}\left\|\Delta^{\prime}\right\|\right|=\Delta^{\prime},(5.3)$ and (5.5) yield $H^{2}\left(\operatorname{spt}\left\|\left(\psi \phi_{n}\right)_{\#} \Delta^{\prime}\right\| \cap B^{3}(p, r)\right) \leq\left(1-\frac{3}{4} k\right)(r / 2) H^{1}(K)$

$$
+5 \zeta r\left[H^{1}(J)-H^{1}(K)\right]+\frac{3}{4} k \pi r^{2} \Theta^{2}(\Delta, p) .
$$

Let us assume without loss of generality

$$
5 \varsigma<\left(1-\frac{3}{4} k\right) \frac{1}{2} \text {. }
$$


Then (5.6) gives us

$$
H^{2}\left(\operatorname{spt}\left\|\left(\psi \phi_{n}\right)_{\#} \Delta^{\prime}\right\| \cap B^{3}(p, r)\right) \leq\left(1-\frac{3}{4} k\right)(r / 2) H^{1}(J)+\frac{3}{4} k \pi r^{2} \Theta^{2}(\Delta, p),
$$

which proves conclusion (2) with $F_{k}^{\prime}=\tilde{\psi} \tilde{\phi}_{n} F_{k}^{3}$ (resp. $\tilde{\psi} \tilde{\phi}_{n} F_{k}^{4}$ ). Conclusion (1) follows from (5.2) and the choice of $\psi, \phi_{n}$.

Finally it remains to prove the lemma with no assumption, i.e., without the assumption that $J$ is piecewise Lipschitz. So $J$ needs to be approximated by a finite number of Lipschitz curves. By Corollary 4, there exists $r_{q}>0$ for each $q \in \sigma_{Y}(\Delta) \cap \partial B^{3}(p, r)$ such that $\sigma_{Y}(\Delta) \cap \partial B^{3}(p, r) \cap B^{3}\left(q, r_{q}\right)=\{q\}$. Let $J_{q}$ be the component of spt $\|\Delta\| \cap \partial B^{3}(p, r) \cap B^{3}\left(q, r_{q}\right)$ containing $q$. Then we replace $J_{q}$ by the union of three geodesic curves $G_{q}^{1} \cup G_{q}^{2} \cup G_{q}^{3}$ in $\partial B^{3}(p, r) \cap B^{3}\left(q, r_{q}\right)$ connecting $q$ to the three points $\left\{q_{1}, q_{2}, q_{3}\right\}=J_{q} \cap \partial B^{3}\left(q, r_{q}\right)$. We assume $r_{q}$ to be appropriately small so that for sufficiently small $\eta>0$,

$$
H^{2}\left(\bigcup_{q} Z_{q}\right)<\eta,
$$

where $Z_{q}$ is the small region on $\partial B^{3}(p, r)$ enclosed by $J_{q}$ and $G_{q}^{1} \cup G_{q}^{2} \cup G_{q}^{3}$, and the union is taken over all $q \in \sigma_{Y}(\Delta) \cap \partial B^{3}(p, r)$. Let $V_{q}^{1}, V_{q}^{2}, V_{q}^{3}$ be open components of $p *\left[\partial B^{3}(p, r) \cap U^{3}\left(q, r_{q}\right) \sim\left(G_{q}^{1} \cup G_{q}^{2} \cup G_{q}^{3}\right)\right] \sim \partial B^{3}(p, r)$. By cutting off filigrees of $\left\{\delta F_{k}\right\}$ (applying Lemma 6 repeatedly at suitable points of spt $\|\Delta\|)$ we can find three open balls $W_{q}^{1}, W_{q}^{2}, W_{q}^{3}$, which are disjoint from $\delta F_{k}$ for all $k$ such that

$$
W_{q}^{l} \cap \partial V_{q}^{l} \approx D, \quad W_{q}^{l} \cap \partial V_{q}^{l} \cap \partial B^{3}(p, r) \cap \partial B^{3}\left(q, r_{q}\right) \neq \varnothing, \quad l=1,2,3 .
$$

Then we apply the replacement argument of Theorem 1 to each $V_{q}^{l}$ and $F_{k}$ with the same alteration as in the second case: For each component $C_{i j}$ of $\delta F_{k} \cap \partial V_{q}^{l}$, define $Y_{i j}$ to be the subset of $\partial V_{q}^{l} \sim \bar{W}_{q}^{l}$ such that $Y_{i j} \approx D$ and $\partial Y_{i j}$ is the outermost Jordan curve of $C_{i j}$ in $\partial V_{q}^{l} \sim \bar{W}_{q}^{l}$. Then, as in Theorem 1, we obtain $F_{k}^{q, 0}\left(=F_{k}\right), F_{k}^{q, 1}, F_{k}^{q, 2}, F_{k}^{q, 3}$ with

$$
\delta F_{k}^{q, l} \sim V_{q}^{l} \subset \delta F_{k}^{q, l-1} \sim V_{q}^{l}, \quad l=1,2,3 .
$$

Using the same arguments as in the second case we can assert that

$$
\operatorname{spt}\left\|\lim _{k \rightarrow \infty}\left|\delta F_{k}^{q, l} \cap V_{q}^{l}\right|\right\| \subset \partial V_{q}^{l}
$$

and that the density of $\lim _{k \rightarrow \infty}\left|\delta F_{k}^{q, l}\right|$ is 1 almost everywhere for each $l=$ $1,2,3$.

Continue the above process for the sequence of points $\{q, \cdots, P\}=\sigma_{Y}(\Delta) \cap$ $\partial B^{3}(p, r)$ until we obtain $\bar{\Delta}=\lim _{k \rightarrow \infty}\left|\delta F_{k}^{P, 3}\right|$. Then we note that the boundary $J^{\prime}$ of spt $\|\bar{\Delta}\| \cap U^{3}(p, r)$ is piecewise Lipschitz. By finding the subset $K^{\prime}$ 
of $J^{\prime}$ which is homeomorphic to $Y \cap \partial B$ (resp. $T \cap \partial B$ ) and using the replacement argument as in the second case above, we can construct a 2-varifold $\hat{\Delta}$ such that $\hat{\Delta}=\lim _{k \rightarrow \infty}\left|\delta \hat{F}_{k}\right|, \delta \hat{F}_{k} \in \Phi, \hat{\Delta}$ has density 1 almost everywhere,

$$
\operatorname{spt}\|\hat{\Delta}\| \sim B^{3}(p, r) \subset \operatorname{spt}\|\Delta\| \sim B^{3}(p, r),
$$

the boundary of spt $\|\hat{\Delta}\| \cap U^{3}(p, r)$ is $K^{\prime}$, and lastly, from (5.8),

$$
H^{2}\left(\operatorname{spt}\|\hat{\Delta}\| \cap \partial B^{3}(p, r)\right) \leq \eta+5 \zeta r\left[H^{1}(J)-H^{1}\left(K^{\prime}\right)\right] .
$$

Hence, as before, we can find diffeomorphisms $\phi_{n}, \psi$ such that

$$
\left\|\left(\psi \phi_{n}\right)_{\#} \hat{\Delta}\right\|\left(U^{3}(p, r)\right) \leq\left(1-\frac{3}{4} k\right)(r / 2) H^{1}\left(K^{\prime}\right)+\frac{3}{4} k \pi r^{2} \Theta^{2}(\Delta, p),
$$

which together with (5.7), (5.9) implies

$$
H^{2}\left(\operatorname{spt}\left\|\left(\psi \phi_{n}\right)_{\#} \hat{\Delta}\right\| \cap B^{3}(p, r)\right) \leq(1-k / 2)(r / 2) H^{1}(J)+(k / 2) \pi r^{2} \Theta^{2}(\Delta, p),
$$

where $\eta$ is absorbed by replacing $\frac{3}{4} k$ by $k / 2$. This completes the proof of Lemma 10.

Recall the diffeomorphism $\nu$ from $B^{3}(p, r) \subset M$ to $B^{3}(0, r) \subset \mathbf{R}^{3}$ which was introduced in $\S 4$. If $M$ is given the Euclidean metric which is pulled back by $\nu$, then one can find a function $\xi(r)=C r^{\alpha}$ with $0 \leq C<\infty$ and $0<\alpha<1 / 3$ such that

$$
H^{2}(\operatorname{spt}\|\Delta\| \cap W) \leq(1+\xi(r)) H^{2}(\operatorname{spt}\|\Gamma\| \cap W),
$$

where $\Gamma$ is as defined in Lemma $10, W=(\operatorname{spt}\|\Delta\| \sim \operatorname{spt}\|\Gamma\|) \cup(\operatorname{spt}\|\Gamma\| \sim$ spt $\|\Delta\|)$ and $r=\operatorname{diam} W$. This property of $\Delta$ is similar to $(\mathbf{M}, \xi, \delta)$ minimality. Therefore we note that the above epiperimetric inequality holds, as $[13$, III.1], for $\Delta$ having this property (not just for area minimizing $\Delta$ ). The following epiperimetric inequality is similar to $[13$, III.1] and slightly weaker than Lemma 10.

Lemma 10'. There exist $0<\varepsilon \leq 1 / 2,0<\varsigma<1 / 100,0<k<\alpha /(1+\alpha)$, and $1<l<\infty$, such that if

(i) $p \in \sigma_{Y}(\Delta)\left(\right.$ resp. $\left.\sigma_{T}(\Delta)\right)$;

(ii) for some $r>0, r^{-2} H^{2}\left(\operatorname{spt}\|\Delta\| \cap B^{3}(p, r)\right) \exp \left(\frac{3}{\alpha} \xi(r)\right)-\pi \Theta^{2}(\Delta, p)<$ $\varepsilon$, and $H D\left(\mu_{1 / r} \tau_{p}\left(\operatorname{spt}\|\Delta\| \cap B^{3}(p, r)\right), \theta Y\right)<\varsigma($ resp., replace $\theta Y$ by $\theta T)$ for some $\theta \in \mathrm{SO}(3)$,

then there exists a 2-varifold $\Gamma$ with $\Gamma=\lim _{k \rightarrow \infty}\left|\delta F_{k}^{\prime}\right|, F_{k}^{\prime} \in \Phi$ such that

(1) $\operatorname{spt}\|\Gamma\| \sim B^{3}(p, r) \subset \operatorname{spt}\|\Delta\| \sim B^{3}(p, r)$ and

(2) $r^{-2} H^{2}\left(\operatorname{spt}\|\Gamma\| \cap B^{3}(p, r)\right) \exp (3 \xi(r) / \alpha)-\pi \Theta^{2}(\Delta, p) \leq(1-k / 2) E(r)+$ $l \xi(r)$, where

$$
E(r)=(2 r)^{-1} H^{1}\left(\mathrm{spt}\|\Delta\| \cap \partial B^{3}(p, r)\right) \exp (3 \xi(r) / \alpha)-\pi \Theta^{2}(\Delta, p) .
$$


Proof. Multiplying conclusion (2) of Lemma 10 by $\exp (3 \xi(r) / \alpha)$, we get

$$
\begin{aligned}
& H^{2}\left(\text { spt }\|\Gamma\| \cap B^{3}(p, r)\right) \exp (3 \xi(r) / \alpha) \\
& \leq(1-k / 2)(r / 2) H^{1}\left(\operatorname{spt}\|\Delta\| \cap \partial B^{3}(p, r)\right) \exp (3 \xi(r) / \alpha) \\
& \quad+(k / 2) \pi r^{2} \theta^{2}(\Delta, p) \exp (3 \xi(r) / \alpha) .
\end{aligned}
$$

The above conclusion (2) is equivalent to

$$
\begin{aligned}
H^{2} \text { (spt } & \left.\|\Gamma\| \cap B^{3}(p, r)\right) \exp (3 \xi(r) / \alpha) \\
\leq & (1-k / 2)(r / 2) H^{1}\left(\operatorname{spt}\|\Delta\| \cap \partial B^{3}(p, r)\right) \exp (3 \xi(r) / \alpha) \\
& +(k / 2) \pi r^{2} \Theta^{2}(\Delta, p)+l r^{2} \xi(r) .
\end{aligned}
$$

Hence it suffices to show

$$
(k / 2) \pi r^{2} \Theta^{2}(\Delta, p) \exp (3 \xi(r) / \alpha) \leq(k / 2) \pi r^{2} \Theta^{2}(\Delta, p)+l r^{2} \xi(r) .
$$

Since $e^{x} \leq 1+A x$ for $A=3^{10}$ and $0 \leq x \leq 10$, we have

$$
\exp (3 \xi(r) / \alpha) \leq 1+A(3 \xi(r) / \alpha) \text { for } 3 / 10 \leq \alpha \leq 1 / 3 \text { and } 0 \leq r \leq 1
$$

Therefore

$$
\begin{aligned}
(k / 2) & \pi r^{2} \Theta^{2}(\Delta, p) \exp (3 \xi(r) / \alpha) \\
& \leq(k / 2) \pi r^{2} \Theta^{2}(\Delta, p)+(k / 2) \pi r^{2} \Theta^{2}(\Delta, p) 3 A \xi(r) / \alpha \\
& \leq(k / 2) \pi r^{2} \Theta^{2}(\Delta, p)+l r^{2} \xi(r)
\end{aligned}
$$

for $l=32 A$, and hence the required result is proved.

Theorem 4 ( $C^{1, \alpha}$ regularity of singular set). (1) At every point $p$ in spt $\|\Delta\|, \Delta$ has a unique tangent cone.

(2) $\sigma_{Y}(\Delta)$ is a one-dimensional $C^{1, \alpha}$ submanifold.

(3) In a neighborhood of $p \in \sigma_{Y}(\Delta)$ (respectively $\sigma_{T}(\Delta)$ ), spt $\|\Delta\|$ is the union of three (respectively six) $C^{1, \alpha / 2}$ manifolds with boundary.

Proof. Chapter IV of [13] proves the above regularity for sets which are $(\mathbf{M}, \xi, \delta)$ minimal under the Lipschitz map. Remember however that competing surfaces of spt $\|\Delta\|$ are not the images of spt $\|\Delta\|$ under Lipschitz maps but are the varifolds which are the limit of $\left|\delta F_{k}\right|$ for some sequence $\left\{F_{k}\right\}$ in $\Phi$. We outline below the alterations to the proof in §IV of [13] that need to be made to accommodate this.

First notice that [13, II.1, II.2, II.4, II.6, and III.1] correspond to Lemma 4, Corollary 2, Lemma 9, Corollary 4, and Lemma $10^{\prime}$ respectively. [13, II.5] holds in our setting if we replace $S_{i}$ by $\mu_{i}(\operatorname{spt}\|\Delta\|)$.

$[13$, IV.1]: Use $(\mathbf{M}, \xi, \delta)$ minimality of spt $\|\Delta\|$ as is described right before Lemma $10^{\prime}$, and then apply Lemma $10^{\prime}$.

[13, IV.4(2)]: Lipschitz maps $f$ and $g$ can be replaced by diffeomorphisms with the same property. 
[13, IV.7(1)]: Lipschitz maps considered here can be replaced by a Lipschitz map $\psi$ which preserves the topology of $F_{k}$, i.e., $M \sim \psi\left(\delta F_{k}\right) \approx B^{\circ}$, $\lim _{k \rightarrow \infty}\left|\delta F_{k}\right|=\Delta$.

Theorem 5. If $M$ is irreducible, then there exists a fundamental domain $F$ in $\Phi$ with least boundary area among all elements of $\Phi$, i.e., $\delta F=\operatorname{spt}\|\Delta\|$.

Proof. In view of Theorem 1. (2) (ii)', it is clear that, for any convex domain $U \subset M$ disjoint from the singular set of spt $\|\Delta\|$, each component of spt $\|\Delta\| \cap U$ is an area minimizing disk. Since spt $\|\Delta\|$ is $C^{1, \alpha / 2}$ up to its singular curve, there exists a $d>0$ such that there is a $C^{1}$ retract $\xi$ of $\Delta_{d} \equiv\{x \in M: \operatorname{dist}(x$, spt $\|\Delta\|)<d\}$ onto spt $\|\Delta\|$. Then we can apply Lemmas 6,7 , and 8 repeatedly at an appropriately chosen set of finite points of spt $\|\Delta\|$ (using a finite open subcover of $M$ ) in such a way that for each $k$ we obtain $F_{k}^{5} \in \Phi$ with

$$
\delta F_{k}^{5} \subset \Delta_{1 / k} \text { and } \lim _{k \rightarrow \infty}\left|\delta F_{k}^{5}\right|=\Delta
$$

Letting $k \rightarrow \infty$, it follows from (5.11) that

$$
i_{*}\left(\pi_{1}(M \sim \operatorname{spt}\|\Delta\|)\right)=0
$$

where $i$ is the inclusion map into $M$. Hence $M \sim \operatorname{spt}\|\Delta\|=p(F)$, or spt $\|\Delta\|=\delta F$ for some fundamental domain $F$ in $\Phi_{0}$. Let $\kappa_{k}$ be a map from $B$ onto $M$ with $\kappa_{k}(\partial B)=\delta F_{k}^{5}$. Then, for $k$ with $1 / k<d, \xi \kappa_{k}$ maps $\partial B$ into spt $\|\Delta\|$. From (5.11) we know that $\xi \kappa_{k}$ is surjective. Now we claim $F$ is in $\Phi$. First suppose $F$ is not connected and a component of $F$ is not homeomorphic to $B^{\circ}$. Then, since $\partial B$ is mapped onto spt $\|\Delta\|$, there exists a connected subset $X$ of spt $\|\Delta\|$ such that two disjoint subsets $X_{1}$ and $X_{2}$ of $\delta F_{k}^{5}$ are mapped onto $X$ by $\xi$. Assume $X$ is the largest such component. If $X$ is a point or a curve, then the tangent cone of $\Delta$ at any point of $X$ cannot be $|D|,|Y|$, or $|T|$. If $X$ is a surface, then the tangent cone at any interior point of $X$ is a plane with density $2(=2|D|)$. Since this contradicts Lemma 9, each component of $M \sim \operatorname{spt}\|\Delta\|$ must be homeomorphic to $B^{\circ}$.

Suppose $M \sim \operatorname{spt}\|\Delta\|=\bigcup_{i=1}^{n} W_{i}, n \geq 2, W_{i} \approx B^{\circ}$, and $W_{i} \cap W_{j}=\varnothing$ if $i \neq j$. Then, using irreducibility of $M$, we paste $W_{i}$ 's to each other in such a way that we can obtain $W \approx B^{\circ}$ with $\bigcup_{i=1}^{n} W_{i} \subset W$ and $\partial W \subset \operatorname{spt}\|\Delta\|$. This is a contradiction since $W$ gives rise to a fundamental domain $\hat{F} \in \Phi$ with $p(\hat{F})=W$ and $H^{2}(\delta \hat{F})<\mathbf{M}(\Delta)$. Hence $M \sim$ spt $\|\Delta\|$ must be homeomorphic to $B^{\circ}$. Clearly $F$ is not reducible. Therefore $F \in \Phi$. 


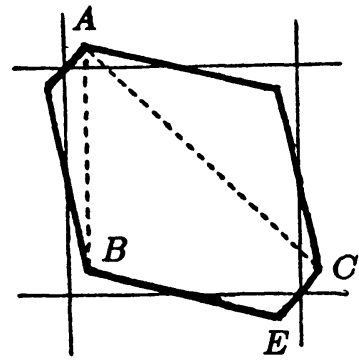

FiguRe 8

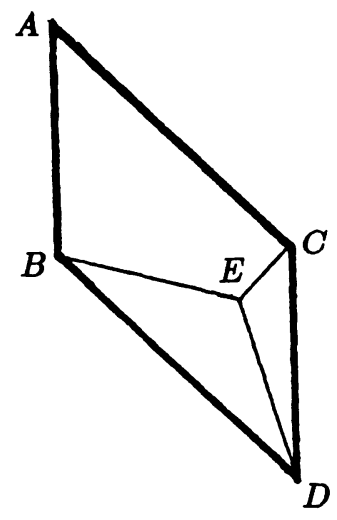

FIGURE 9

\section{Appendix}

1. Given a flat $\mathbf{T}^{2}$, not necessarily a square torus, we know from the proposition of $\S 4$ that a hexagon is a minimizing fundamental domain of the $\mathbf{T}^{2}$. Cut this hexagon along the dotted lines $A B$ and $A C$ (Figure 8).

After translating and pasting, we get another fundamental domain which is a parallelogram $A B C D$ (Figure 9). Note that the boundary length of the minimizing hexagon is twice the sum of the lengths of $E B, E C, E D$, and that $E$ is a triple point joining the vertices of the acute triangle $B C D$. Since there exists one and only one triple point in an acute triangle we conclude that there exists a unique minimizing fundamental domain of flat $\mathbf{T}^{2}$ up to translations.

2. The standard projection map $\pi$ from $S^{2} \times S^{1}$ onto $S^{2}$ is an areadecreasing map: for any $H^{2}$-measurable set $X \subset S^{2} \times S^{1}$,

$$
H^{2}(X) \geq H^{2}(\pi(X))
$$

Moreover, for any fundamental domain $F$ of $S^{2} \times S^{1}$ in $\Phi_{0}, \pi$ maps $p(\partial F)$ onto $S^{2}$. Hence

$$
H^{2}(p(\partial F)) \geq H^{2}(\pi(p(\partial F)))=H^{2}\left(S^{2}\right)=H^{2}\left(p\left(\partial\left(S^{2} \times(0,1)\right)\right)\right) .
$$

Thus $S^{2} \times(0,1)$ is a minimizing fundamental domain of $S^{2} \times S^{1}$.

3. 100 years ago, Sir William Thomson [14] considered a similar problem, periodic minimal partitioning of $\mathbf{R}^{3}$. His construction gives a candidate for the periodic division of $\mathbf{R}^{3}$ with minimum partitional area. But it has never been proved that his partitioning is the minimum. This candidate is a 14faced domain whose boundary consists of six quadrilateral faces and eight hexagonal faces. This domain can be roughly obtained by truncating all 
vertices of regular octahedron. Here quadrilateral faces are flat, hexagonal faces are a monkey saddle, and each edge is a plane curve. The faces meet with the correct $120^{\circ}$ angles along the edges and with the correct tetrahedral angles at the vertices. It turns out that Thomson's domain is a fundamental domain of a skew torus which is spanned by $4^{-1 / 3}(2,0,0), 4^{-1 / 3}(0,2,0)$, and $4^{-1 / 3}(1,1,1)$. We should mention that $R$. Kusner showed 14 is a lower bound for the number of faces of a minimizing fundamental domain of any flat torus. Notice that minimal partitioning of $\mathbf{R}^{3}$ is more general than periodic minimal partitioning of $\mathbf{R}^{3}$, and these two partitioning problems are more general than the problem of finding a minimizing fundamental domain of a specific flat torus.

\section{References}

[1.] W. K. Allard, On the first variation of a varifold, Ann. of Math. (2) 95 (1972) 417-491.

[2.] W. K. Allard \& F. J. Almgren, Jr., The structure of stationary one dimensional manifolds with positive density, Invent. Math. 34 (1976) 83-97.

[3.] F. J. Almgren, Jr., Existence and regularity almost everywhere of solutions to elliptic variational problems with constraints, Mem. Amer. Math. Soc. No. 165, 1976.

[4.] F. J. Almgren, Jr. \& L. Simon, Existence of embedded solutions of Plateau's problem, Ann. Scoula Norm. Sup. Pisa Cl. Sci. (4) 6 (1979) 447-495.

[5.] C. B. Croke, Some isoperimetric inequalities and eigenvalue estimates, Ann. Sci. École Norm. Sup. (4) 13 (1980) 419-435.

[6.] E. De Giorgi, Nuovi teoremi relativi alle misure $(r-1)$-dimensionali inn uno spazio ad $r$ dimensioni, Ricerche Mat. 4 (1955) 95-113.

[7.] H. Federer, Geometric measure theory, Springer, Berlin, 1969.

[8.] D. Kinderlehrer, L. Nirenberg \& J. Spruck, Régularité dans les problèmes elliptiques á frontière libre, C. R. Acad. Sci. Paris Sér. A-B 286 (1978) A1187-A1190.

[9.] W. Meeks III, L. Simon \& S.-T. Yau, Embedded minimal surfaces, exotic spheres, and manifolds with positive Ricci curvature, Ann. of Math. (2) 116 (1982) 621-659.

[10.] E. R. Reifenberg, Solution of the Plateau problem for m-dimensional surfaces of varying topological type, Acta Math. 104 (1960) 1-92.

[11.] L. Simon, Lectures on geometric measure theory, Australian National University, 1984.

[12.] J. E. Taylor, Regularity of the singular sets of two dimensional area-minimizing flat chains modulo 3 in $\mathbf{R}^{3}$, Invent. Math. 22 (1973) 119-139.

[13.] __ The structure of singularities in soap-bubble-like and soap-film-like minimal surfaces, Ann. of Math. (2) 103 (1976) 489-539.

[14.] W. Thomson, On the division of space with minimum partitional area, Acta Math. 11 (1888) 121-134. 
